\title{
Impaired replication elongation in Tetrahymena mutants deficient in histone H3 Lys 27 monomethylation
}

\author{
Shan Gao, ${ }^{1,10}$ Jie Xiong, ${ }^{2,3,10}$ Chunchao Zhang, ${ }^{4}$ Brian R. Berquist, ${ }^{5}$ Rendong Yang, ${ }^{6}$ Meng Zhao, ${ }^{6}$ \\ Anthony J. Molascon, ${ }^{1}$ Shaina Y. Kwiatkowski, ${ }^{1}$ Dongxia Yuan, ${ }^{2}$ Zhaohui Qin, ${ }^{6,7}$ Jianfan Wen, ${ }^{3}$ \\ Geoffrey M. Kapler, ${ }^{5}$ Philip C. Andrews, ${ }^{4,8,9}$ Wei Miao, ${ }^{2,11}$ and Yifan Liu ${ }^{1,11}$ \\ ${ }^{1}$ Department of Pathology, University of Michigan, Ann Arbor, Michigan 48109, USA; ${ }^{2}$ Key Laboratory of Aquatic Biodiversity \\ and Conservation, Institute of Hydrobiology, Chinese Academy of Sciences, Wuhan 430072, China; ${ }^{3}$ State Key Laboratory \\ of Genetic Resources and Evolution, Kunming Institute of Zoology, Chinese Academy of Sciences, China; ${ }^{4}$ Department \\ of Computational Medicine and Bioinformatics, University of Michigan, Ann Arbor, Michigan 48109, USA; ${ }^{5}$ Department of \\ Molecular and Cellular Medicine, College of Medicine, Texas A\&M Health Science Center, College Station, Texas 77843, USA; \\ ${ }^{6}$ Department of Biostatistics and Bioinformatics, Emory University, Atlanta, Georgia 30322, USA; ${ }^{7}$ Center for Comprehensive \\ Informatics, Emory University, Atlanta, Georgia 30322, USA; ${ }^{8}$ Department of Chemistry, University of Michigan, Ann Arbor, \\ Michigan 48109, USA ${ }^{9}$ Department of Biological Chemistry, University of Michigan, Ann Arbor, Michigan 48109, USA
}

Replication of nuclear DNA occurs in the context of chromatin and is influenced by histone modifications. In the ciliate Tetrahymena thermophila, we identified TXR1, encoding a histone methyltransferase. TXR1 deletion resulted in severe DNA replication stress, manifested by the accumulation of ssDNA, production of aberrant replication intermediates, and activation of robust DNA damage responses. Paired-end Illumina sequencing of ssDNA revealed intergenic regions, including replication origins, as hot spots for replication stress in $\triangle T X R 1$ cells. $\triangle T X R 1$ cells showed a deficiency in histone H3 Lys 27 monomethylation (H3K27me1), while $\triangle E Z L 2$ cells, deleting a Drosophila $E(z)$ homolog, were deficient in $\mathrm{H} 3 \mathrm{~K} 27$ di- and trimethylation, with no detectable replication stress. A point mutation in histone $\mathrm{H} 3$ at Lys 27 (H3 K27Q) mirrored the phenotype of $\triangle T X R 1$, corroborating H3K27me1 as a key player in DNA replication. Additionally, we demonstrated interactions between TXR1 and proliferating cell nuclear antigen (PCNA). These findings support a conserved pathway through which H3K27me1 facilitates replication elongation.

[Keywords: histone methyltransferase; H3 Lys 27 methylation; replication stress; replication elongation; ssDNA; replication origin]

Supplemental material is available for this article.

Received April 2, 2013; revised version accepted July 1, 2013.

Propagating genetic information with high fidelity is a central challenge in all forms of life. DNA is particularly vulnerable during replication, a process that disrupts and then restores the structural integrity of the DNA double helix. DNA replication of large eukaryotic genomes begins at multiple replication origins, which are licensed by binding of the origin recognition complex (ORC) (Mechali 2010). DNA replication is initiated as the CDC45, MCM27, and GINS DNA helicase complex (CMG), assembled at a replication origin, is activated and unwinds template DNA (Tanaka and Araki 2010). This is followed by the synthesis of nascent strands catalyzed by DNA polymerases and

\footnotetext{
${ }^{10}$ These authors contributed equally to this work.

${ }^{11}$ Corresponding authors

E-mail yifan@med.umich.edu

E-mail miaowei@ihb.ac.cn

Article published online ahead of print. Article and publication date are online at http://www.genesdev.org/cgi/doi/10.1101/gad.218966.113.
}

elongation of two diverging replication forks emanating from a replication origin (Sclafani and Holzen 2007). Active replication forks are characterized by dynamic association with various DNA polymerases, helicases, nucleases, and ligases as well as important accessory factors like the replication protein A (RPA) complex and proliferating cell nuclear antigen (PCNA), which together, as a functional entity, are often referred to as the replisome (MacNeill 2012). Replication elongation can be hindered by metabolic changes affecting the free nucleotide pool, various damages to the DNA template, and perturbations to the replisome (Aguilera and Gomez-Gonzalez 2008). These kinds of replication stress can slow and stall replication forks, even leading to double-strand breaks (DSBs) (Branzei and Foiani 2010). Consequently, cells mobilize the DNA damage response (DDR), a collective of conserved pathways that sense assault on DNA, transduce the signals, and elicit downstream events, including 
DNA repair and transcriptional responses (Harper and Elledge 2007; Polo and Jackson 2011). All of these can stabilize replication forks, restart stalled ones, and repair broken ones (Branzei and Foiani 2010). Nonetheless, replication stress and inappropriately repaired replication forks are a major source of DNA mutation, implicated in many genetic diseases as well as cancer (Friedberg et al. 2006; Weinert et al. 2009; Malkova and Haber 2012).

In eukaryotic cells, nuclear DNA is packaged into chromatin, of which the nucleosome is the most basic unit, formed by $\sim 200$ base pairs (bp) of DNA wrapped around a protein core of histones (Kornberg 1974). In addition to genetic information carried by DNA, a large amount of epigenetic information is carried by chromatin, mainly in the form of various post-translational modifications (PTMs) of histones (Strahl and Allis 2000; Jenuwein and Allis 2001). Replication of nuclear DNA is tightly coupled with histone deposition and nucleosome assembly (Groth et al. 2007; Avvakumov et al. 2011). Various histone-modifying enzymes, including acetyltransferases, deacetylases, and methyltransferases, play important roles in DNA replication (Groth et al. 2007; Avvakumov et al. 2011). Histone modifications have been shown to regulate replication licensing (Miotto and Struhl 2010; Tardat et al. 2010), control replication timing (Vogelauer et al. 2002), and prevent rereplication (Jacob et al. 2010; Tardat et al. 2010). Nonetheless, we have only a limited understanding of how the duplication of genetic and epigenetic information is coordinated, as epigenetic factors affecting replication elongation have so far been poorly characterized.

Tetrahymena thermophila is a well-established ciliate model organism for epigenetic studies (Meyer and Chalker 2007). Like other ciliates, Tetrahymena contains within the same cytoplasmic compartment two structurally and functionally differentiated nuclei: the macronucleus and the micronucleus (Karrer 2012). An alternative cell cycle is present in macronuclei: After DNA replication, instead of going through mitosis characterized by chromatin condensation and spindle formation, polyploid macronuclei randomly distribute their contents in a nuclear division process referred to as amitosis. This unique biology facilitates biochemical and cytological analyses of DNA replication in macronuclei, particularly the highly amplified rDNA minichromosome (Cech and Brehm 1981; MacAlpine et al. 1997; Zhang et al. 1997; Donti et al. 2009).

We recently identified in Tetrahymena a gene encoding the sole homolog to Arabidopsis ATXR5 and ATXR6 (Raynaud et al. 2006; Jacob et al. 2009, 2010), referred to as TXR1. Like ATXR5 and ATXR6, TXR1 features a SET domain, conferring the histone methyltransferase (HMT) activity (Jacob et al. 2009), as well as a PCNA-interacting protein (PIP) box, potentially important for its recruitment to active replication sites (Raynaud et al. 2006). In Arabidopsis, characterization of the atxr5/atxr6 hypomorphic mutant implicated them in histone H3 Lys 27 monomethylation (H3K27me1), heterochromatin formation, and replication control (Jacob et al. 2009, 2010). Here, we provide detailed phenotypic analysis of $\triangle T X R 1$ cells, which unexpectedly showed severe replication stress, supported by accumulation of ssDNA, production of aberrant replication intermediates (RIs), and activation of robust DDRs. Furthermore, paired-end Illumina sequencing of ssDNA revealed that they were specifically enriched in intergenic regions, including known replication origins. Quantitative mass spectrometry (MS) analysis of global histone modification patterns revealed that $\triangle T X R 1$ cells were deficient in $\mathrm{H} 3 \mathrm{~K} 27 \mathrm{me}$. The phenotype was in strong contrast to $\triangle E Z L 2$ cells, which were deficient in H3K27 dimethylation (H3K27me2) and H3K27 trimethylation (H3K27me3), and showed no replication stress. The H3 K27Q mutant mirrored the phenotype of $\triangle T X R 1$, corroborating $\mathrm{H} 3 \mathrm{~K} 27 \mathrm{mel}$ as a key player in DNA replication. Additionally, we demonstrated physical and functional interactions between TXR1 and PCNA, probably involved in recruiting TXR1 to active replication forks. The results shed light on a pathway through which epigenetic factors can impact replication elongation.

\section{Results}

Accumulation of ssDNA in $\triangle T X R 1$ cells

We identified in the Tetrahymena genome the sole homo$\log$ to Arabidopsis ATXR5 and ATXR6, referred to as TXR1, and generated the corresponding deletion strain $\triangle T X R 1$ (Supplemental Figs. 1, 12). We observed accumulation of ssDNA in $\triangle T X R 1$, but not wild-type, cells as 5-bromo-2'deoxyuridine (BrdU) immunofluorescence signals detected under nondenaturing conditions (Fig. 1A). For the incorporated $\mathrm{BrdU}$ to be accessible to its cognate antibody, DNA must be in single-stranded forms, which in normal cells are generated in a significant amount only after acid or nuclease treatment. The BrdU signals were detected only in S-phase and early G2-phase cells immediately after pulse-labeling (Fig. 1B). This result strongly suggests that these ssDNA were derived from nascent strands of DNA synthesis, in support of replication stress in $\triangle T X R 1$ cells. Consistent with this interpretation, $\triangle T X R 1$ cells grew significantly slower than wild-type cells, which could be attributed mostly to prolonged $S$ phase (Supplemental Fig. 2A, B). ssDNA distribution during cell cycle progression was followed by BrdU pulse-chase (Fig. 1A): In S-phase and early G2-phase $\triangle T X R 1$ cells, the BrdU signals entirely covered macronuclei, with a granulated pattern of distribution suggestive of replication foci; in mid and late G2-phase cells, the BrdU signals diminished in intensity as well as distribution and were eventually sequestered in a few foci at the center of macronuclei; the BrdU signals often retreated to a single focus residing in a chunk of DNA left between two divided macronuclei during amitosis, giving rise to a chromatin extrusion body (CEB) (Salamone and Nachtwey 1979). Counting these staged cells revealed a temporal wave of redistribution accompanying cell cycle progression (Fig. 1B), consistent with transfer of ssDNA from S-phase macronuclei to CEBs. CEBs are likely the equivalent to mammalian micronuclei induced by replication stress and genotoxic reagents (Fenech et al. 2011). These CEBs were significantly overrepresented in $\triangle T X R 1$ cells (Supplemental Fig. 2C), similar 
A

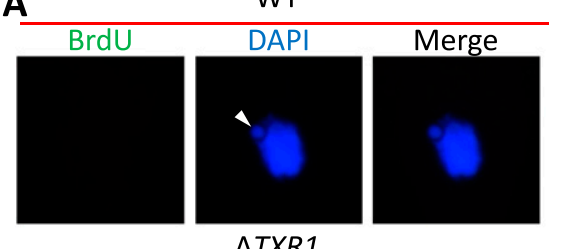

$\Delta T X R 1$

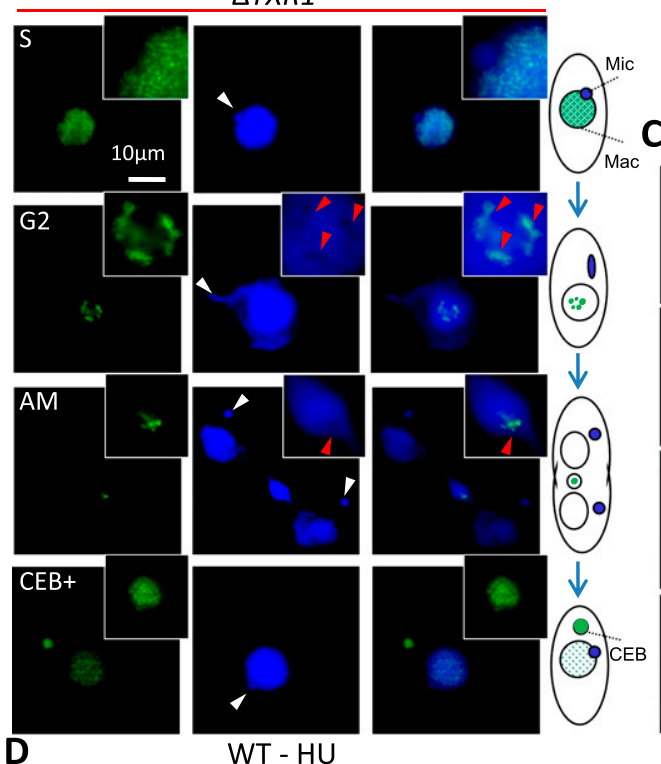

D

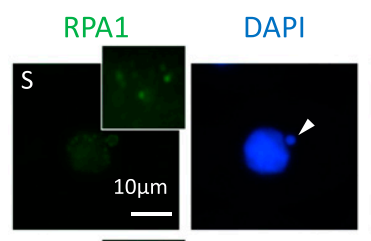

G2
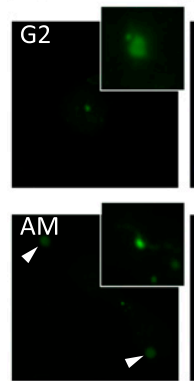
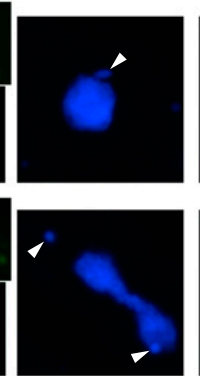
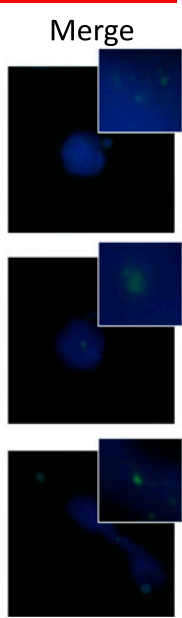

B
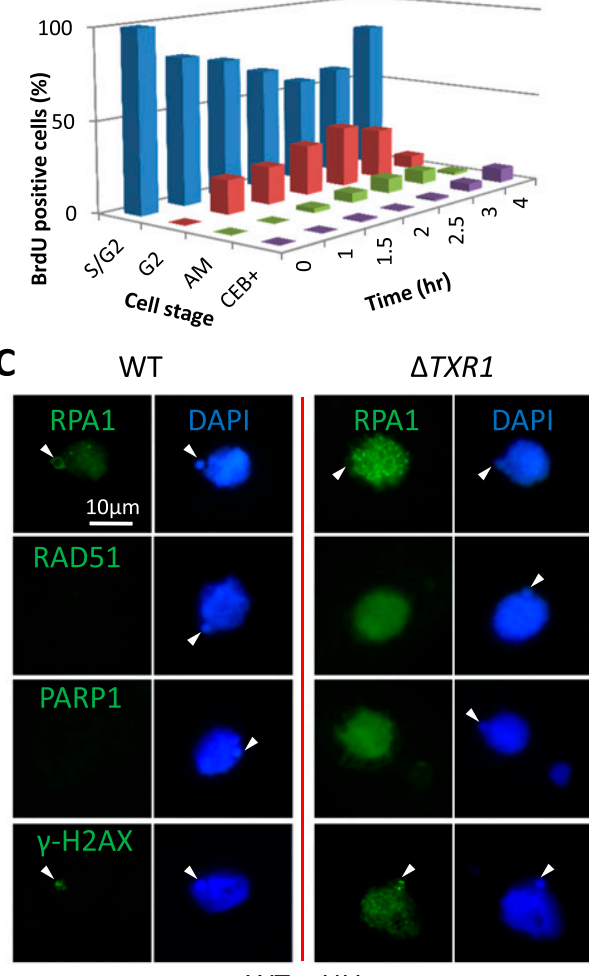

$\mathrm{WT}+\mathrm{HU}$

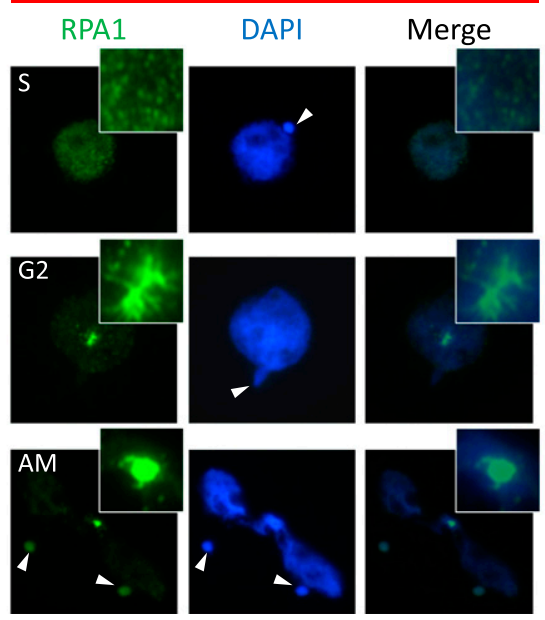

Figure 1. Accumulation of ssDNA in $\triangle T X R 1$ and HU-treated wild-type (WT) cells. (A) Cell cycle-dependent distribution of ssDNA in $\triangle T X R 1$ cells after BrdU pulse-chase. For pulse-labeling, cells were incubated for 30 min with $0.4 \mathrm{mM}$ BrdU in SPP medium and immediately fixed for immunofluorescence staining. For the chase experiment, pulse-labeled cells were washed twice before being resuspended with fresh SPP medium, and time points were taken at half-hour intervals for $4 \mathrm{~h}$. (S) S and early G2 phase; (G2) mid and late $\mathrm{G} 2$ phase; (AM) amitosis; (CEB+) BrdU staining in CEBs. (White arrowheads) Micronuclei; (red arrowheads) macronuclear regions poorly stained with DAPI. Note that the cell cycle-dependent redistribution of ssDNA does not reflect the temporal replication program as described in mammalian cells (O'Keefe et al. 1992). (B) Quantification of cell cycle distribution of BrdU-positive $\Delta T X R 1$ cells. Details for cell cycle staging are described in the Supplemental Material. $(C)$ Induction of RPA1, RAD51, PARP1, and $\gamma$-H2A.X in $\triangle T X R 1$ cells. Note the accumulation of RPA1 and $\gamma$-H2A.X in micronuclei (white arrowheads) as well as in macronuclei. $(D)$ Accumulation of RPAl in HU-treated wild-type cells. Cells were incubated for $20 \mathrm{~h}$ with $5 \mathrm{mM} \mathrm{HU}$ in SPP medium before being fixed for immunofluorescence staining. Note the similarity in abnormal nuclear morphology of $\Delta T X R 1$ and HU-treated cells.

to what has been observed in Tetrahymena cells treated with DNA replication inhibitors like aphidicolin and hydroxyurea (HU) (Yakisich et al. 2006; Kaczanowski and Kiersnowska 2011). DNA was eventually degraded in CEBs
(Supplemental Fig. 2D), supporting their involvement in the disposal of aberrant DNA generated during replication.

We also observed accumulation of ssDNA-binding/ sensing proteins in $\triangle T X R 1$ cells (Fig. 1C). The Tetrahymena 
homolog of RPA1, the largest subunit of the canonical eukaryotic ssDNA-binding complex (Broderick et al. 2010), was highly induced at mRNA and protein levels (Figs. 1C, 5C [below]; Supplemental Fig. 3A). Cell fractionation revealed accumulation of RPA1 in the chromatin-bound as well as the soluble fraction (Supplemental Fig. 3B). Chromatin immunoprecipitation demonstrated increased RPA1 binding to chromatin in $\triangle T X R 1$ cells (Supplemental Fig. 3C). Similar induction was observed with the Tetrahymena homologs of PARP1 and other poly-ADP-ribose metabolism enzymes (Figs. 1C, 5C [below]; Supplemental Fig. 3B) involved in sensing and repairing single-strand breaks (SSBs) (Luo and Kraus 2012) as well as RAD51 (Fig. 1C, 5C [below]; Supplemental Fig. 3B), the ssDNA-binding recombinase involved in homologous recombination (HR) (Masson and West 2001). There was also a huge increase in $\gamma$-H2A.X levels (Fig. 1C) as well as transcriptional inductions of Tetrahymena homologs of MRE11, ATR, and BRCT domain proteins (Fig. 5C, below), indicative of ATR pathway activation (Flynn and Zou 2011). All of these signals peaked in S-phase macronuclei, providing additional support for generation of excess ssDNA during replication.

In $\triangle T X R 1$ cells, ssDNA-enriched regions were similarly marked as foci of BrdU, $\gamma$-H2A.X, and RPA1, in the order of increasing sensitivity for detection (Fig. 1; Supplemental Fig. 4). The $\gamma$-H2A.X and RPA1 signals exhibited cell cycle-dependent redistribution similar to BrdU (Supplemental Fig. 4), in agreement with ATR activation by ssDNA accumulation and RPA binding (Zou and Elledge 2003). RPA1 foci, much smaller and fewer, were detected even in wild-type cells (Fig. 1D). HU treatment of wildtype cells, depleting the dNTP pool and impeding replication elongation (Reichard 1988), dramatically increased the size and number of RPA1 foci (Fig. 1D), accompanied by a dramatic increase of $\gamma$-H2A.X levels (Supplemental Fig. 5) and induction of transcriptional responses similar to that of $\triangle T X R 1$ cells (Fig. 5D, below). In all, this argues that there is severe replication stress in $\triangle T X R 1$ cells, resembling that induced by HU treatment.

\section{Accumulation of RIs in $\triangle T X R 1$ cells}

Replication stress is often accompanied by DNA SSBs or DSBs (Branzei and Foiani 2010). To test their presence, we analyzed genomic DNA from $\triangle T X R 1$ and wild-type cells by alkaline (for SSBs) and neutral (for DSBs) agarose gel electrophoresis (Fig. 2A-C). Most of the genomic DNA isolated from growing Tetrahymena cells are from polyploid macronuclei, containing $\sim 200$ different chromosomes averaging a few hundred kilobases in size (Eisen et al. 2006; Coyne et al. 2008). Under both conditions, intact chromosomes were detected as high-molecular-weight smears (Fig. 2A-C). Under alkaline conditions, immunodetection of pulse-labeled BrdU as well as ethidium bromide staining revealed low-molecular-weight smears, representing short nascent strands (Fig. 2A). The lowmolecular smear was more abundant and smaller in size in $\triangle T X R 1$ cells than in wild-type cells (Fig. 2A). In the chase experiments, the low-molecular-weight smear quickly shifted upward to high-molecular-weight regions in wild-type cells, while this shift was delayed in $\triangle T X R 1$ cells (Fig. 2A). Under neutral conditions, any DNA molecules smaller in size than the 21-kb rDNA minichromosome are DSB-generated fragments, which were apparent in $\triangle T X R 1$ but not wild-type cells (Fig. 2B,C). Pulse-labeled BrdU was detected in these DNA fragments, with the signals diminishing during the chase period, indicative of DSB generation in S phase (Fig. 2C).

BrdU pulse-labeling revealed that the bulk of newly synthesized DNA was retained in the sample plug of the neutral gel (Fig. 2B), consistent with replication bubbles being trapped by agarose (Mesner et al. 2006). In wild-type cells, BrdU signals in the sample plug quickly diminished in the chase experiment, shifting to the high-molecularweight smear representing finished replication products; on the contrary, this shift of BrdU signals was delayed in $\Delta$ TXR1 cells (Fig. 2B). This accumulation of RIs is consistent with slowing or stalling of replication forks.

To further study the replication defects in $\triangle T X R 1$ cells, we used two-dimensional (2D) neutral-neutral agarose gel electrophoresis (2D gel) to visualize RIs in the highly amplified and well-characterized rDNA minichromosome (Fig. 2D). HindIII digestion releases an rDNA palindromic fragment (Fig. 3A), each half featuring a replication origincontaining region derived from an ancient direct duplication event (Amin and Pearlman 1985; MacAlpine et al. 1997). Probably due to their close proximity, only one replication origin is activated in any given rDNA molecule (Cech and Brehm 1981; MacAlpine et al. 1997). This generates a $2 \mathrm{D}$ gel pattern of a bubble arc transitioning to a descending Y arc, punctuated by RI accumulation at three specific pausing sites (MacAlpine et al. 1997). In $\triangle T X R 1$ cells, this overall pattern was maintained, indicative of normal replication initiation; however, it was superimposed with two aberrant RIs evident in 2D gels (Fig. 2D). First, there were signals proximal to the track of the hypothetical ascending $\mathrm{Y}$ arc (RI-1). They can be attributed to Y-like molecules derived from stalled replication bubbles by a DSB at forks (Lopes et al. 2001; Doksani et al. 2009). This interpretation is compatible with the neutral agarose gel result indicative of DSBs in the newly synthesized DNA (Fig. 2C). Intriguingly, benzoylatednaphthoylated DEAE (BND) cellulose, which preferentially captures ssDNA, specifically enriched the Y-like molecules (RI-1). This implicates them as the ssDNA-containing species accumulating in $\triangle T X R 1$ cells and suggests fork stalling as the molecular mechanism underlying the replication stress and elongation defects. Second, there was a pronounced vertical smear intersecting the descending $\mathrm{Y}$ arc at the $2 \mathrm{n}$ position (RI-2). This pattern, typically defined as an $\mathrm{X}$ spike, is diagnostic for branchmigrating recombination intermediates formed readily upon replication stress (Lopes et al. 2003; Liberi et al. 2005; Minca and Kowalski 2010, 2011). Intriguingly, in 2D gels, after BND cellulose capturing of RIs, the $\mathrm{X}$ spike was eliminated. This result strongly suggests that the $\mathrm{X}$ spike represents joint molecules with Holliday junctions, which are ssDNA-poor (Minca and Kowalski 2011), but not hemicatenanes, which are ssDNA-rich (Liberi et al. 2005). After BND cellulose capturing, we also detected 




B

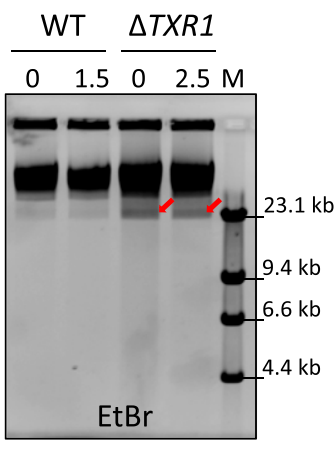

C
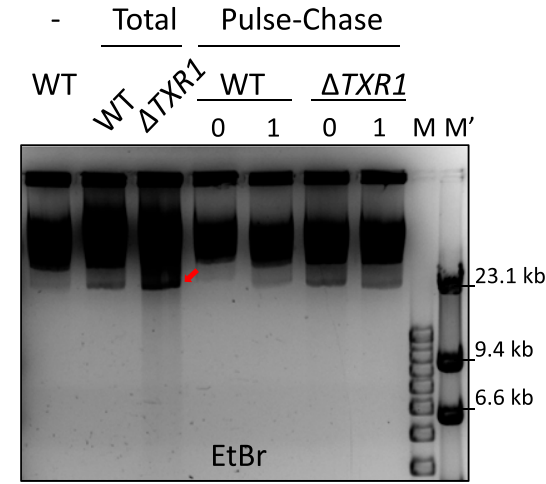

D
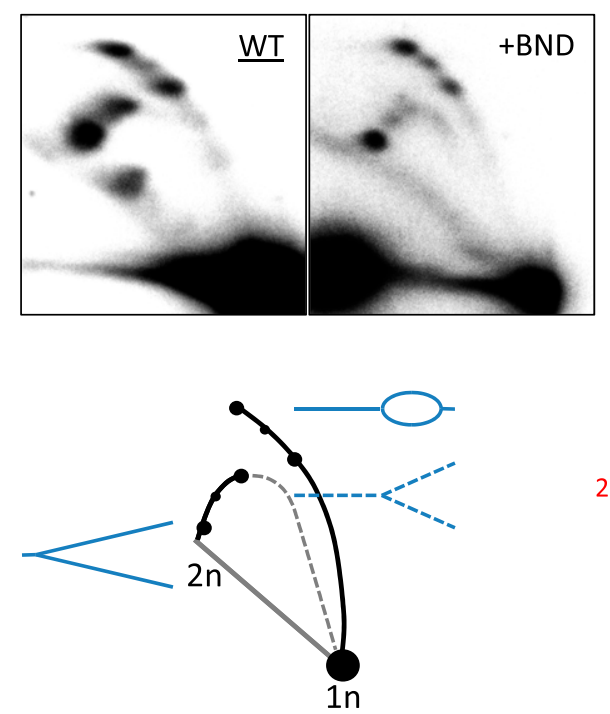
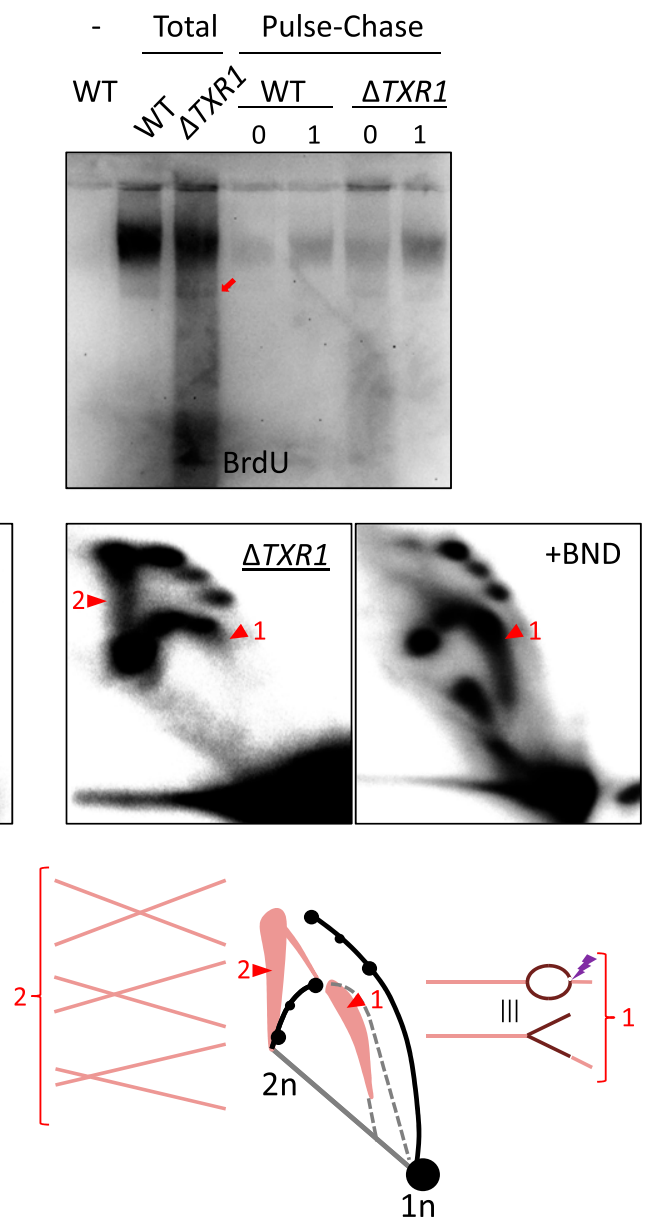

Figure 2. Accumulation of RIs in $\triangle T X R 1$ cells. (A) Accumulation of short nascent strands in $\triangle T X R 1$ cells, revealed by BrdU pulsechase and alkaline agarose gel electrophoresis. For detecting incorporated BrdU, DNA was transferred to $\mathrm{Nylon}^{+}$membrane and probed by $\alpha$-BrdU antibody. (Numbers) Time (in hours) after BrdU pulse-labeling; ( $M$ and $M^{\prime}$ ) DNA markers; (EtBr) ethidium bromide staining; (BrdU) BrdU immunoblotting. $(B)$ Accumulation of RIs in $\triangle T X R 1$ cells, revealed by BrdU pulse-chase and neutral agarose gel electrophoresis. (Red arrows) The rDNA minichromosome; (dashed arrows) shift from RIs to finished products. (C) Accumulation of DSBs in $\triangle T X R 1$ cells, revealed by BrdU pulse-chase and neutral agarose gel electrophoresis. DNA was in-gel-trapped (Khan and Nawaz 2007) and probed by $\alpha$-BrdU antibody. (D) Accumulation of aberrant RIs (red arrowheads) in $\Delta T X R 1$ cells, revealed by $2 \mathrm{D}$ neutralneutral agarose gel electrophoresis. (+BND) RI enrichment by BND cellulose prior to the $2 \mathrm{D}$ gel. Illustrated in the schematics are the interpretations of normal RIs in wild-type (WT) cells and aberrant RI-1 and RI-2 in $\triangle T X R 1$ cells.

a signal connecting the $\mathrm{Y}$ arc with the apex of the $\mathrm{X}$ spike, supporting that the stalled forks are restarted by HR. We conclude that the 2D gel pattern of $\triangle T X R 1$ cells deviates dramatically from wild-type cells, manifested as stalled and even broken replication forks, which are subsequently restarted by HR.

\section{Distribution of ssDNA in the rDNA minichromosome} in $\triangle T X R 1$ cells

We identified ssDNA-representing read pairs in pairedend Illumina sequencing of genomic DNA /see the Supplemental Material for details), which were much 

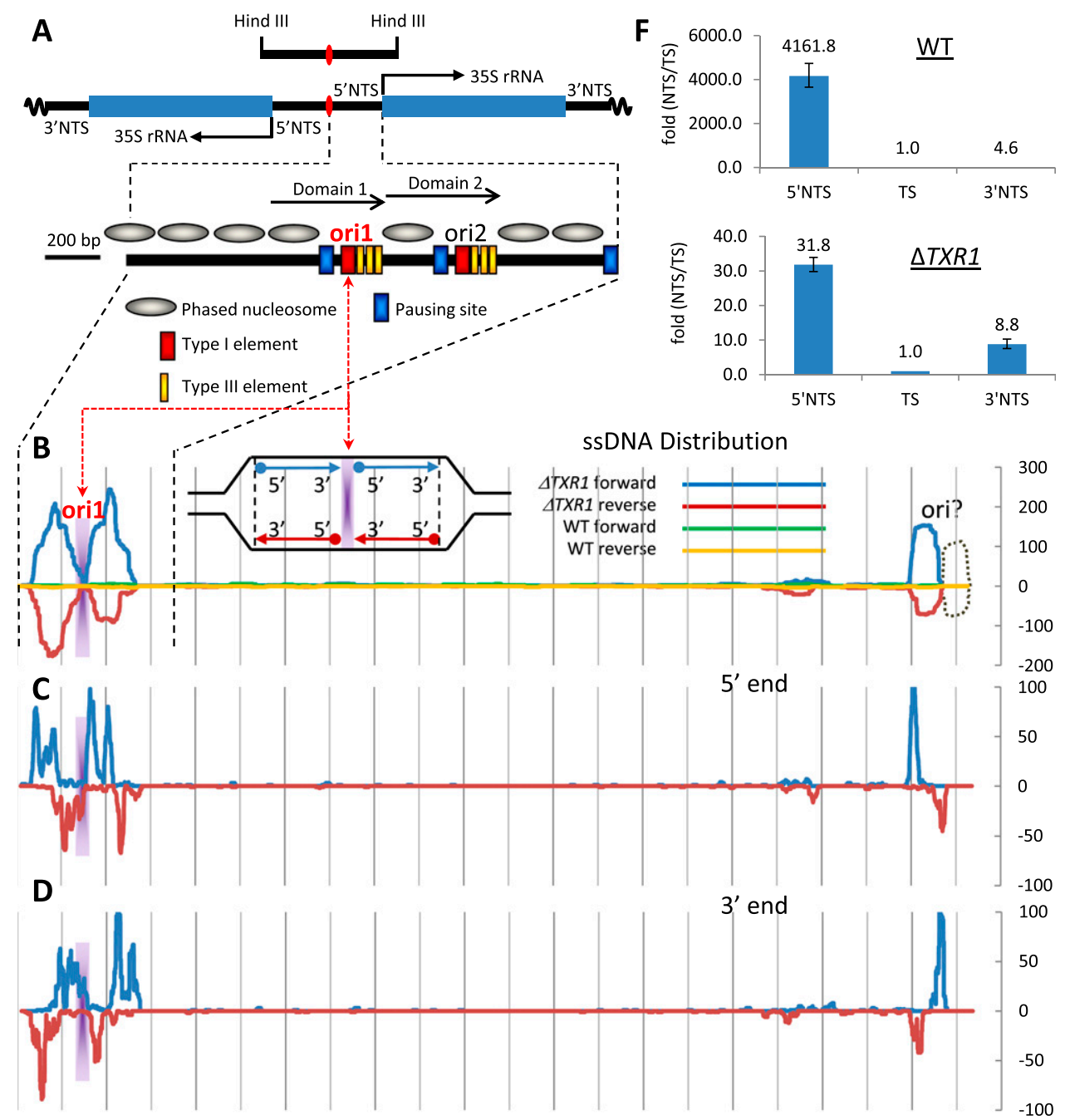

E Coverage Ratio

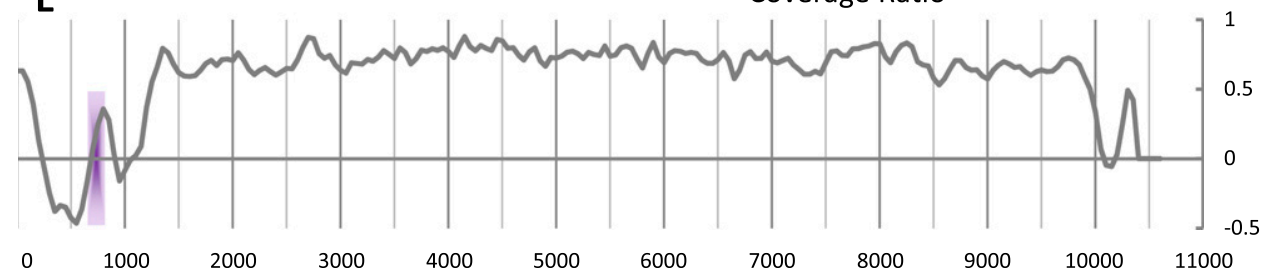

Figure 3. Distribution of ssDNA in the rDNA minichromosome in $\triangle T X R 1$ cells. $(A)$ Schematic of the palindromic rDNA minichromosome, illustrating the transcribed region, the $5^{\prime} \mathrm{NTS}$, the $3^{\prime}$ NTS, telomeres, and the palindromic HindIII fragment containing replication origins. Details of the $5^{\prime}$ NTS: replication origins (ori1 and ori2), phased nucleosomes, and DNA elements (pausing sites and type I/III elements). (B) Distribution of ssDNA in the rDNA from wild-type (WT) and $\Delta T X R 1$ cells in both forward and reverse directions. The $Y$-axes in $B-D$ represent abundance of the ssDNA reads. Note the symmetry around oril and its correspondence to the schematic of nascent strands in a replication bubble. $(C, D)$ Distribution of $5^{\prime}(C)$ and $3^{\prime}(D)$ ends of the ssDNA from $\triangle T X R 1$ cells. $(E)$ Illumina sequencing coverage ratio $\left(\log _{2}\right)$ of $\triangle T X R 1$ and wild-type cells, calculated along the length of the rDNA (details provided in the Supplemental Material). The ratio is based on the abundance of total mappable reads, which are predominantly dsDNA. Note its strong negative correlation with the ssDNA abundance in $\triangle T X R 1$ cells. $(F)$ Validation of rDNA replication origins by analysis of short nascent strands (SNSs). Note the enrichment in the rDNA 3' NTS in $\triangle T X R 1$ cells, normalized against SNS levels in the rDNA transcribed region (TS). The difference in fold of enrichment is due to the higher background of ssDNA in $\Delta T X R 1$ cells.

more abundant in $\triangle T X R 1$ than wild-type cells (Fig. 3B; Supplemental Figs. 6-8). They manifested as apparent inverted duplications formed by microhomology-mediated fold back of ssDNA and self-templated synthesis (Supplemental Figs. 7, 8). These read pairs were further distinguished by having the same initial sequence from both read 1 and read 2, resulting from the end repair process during the sample preparation for Illumina sequencing (Supplemental Figs. 6, 7). Importantly, this approach for ssDNA sequencing, which has been imple- 
mented to map meiotic recombination hot spots (Khil et al. 2012), allowed us to determine the genome-wide distribution of the ssDNA accumulated in $\triangle T X R 1$ cells.

In Tetrahymena, the rDNA minichromosome is greatly amplified in the macronucleus ( $\sim 9000$ copies, compared with $\sim 45$ copies for non-rDNA chromosomes) (Engberg and Pearlman 1972; Yao et al. 1974), enabling ultradeep sequencing coverage $(\sim 4000 \times$, compared with $\sim 20 \times$ for non-rDNA chromosomes). We recovered 1144 rDNAspecific ssDNA in the $\triangle T X R 1$ sample /compared with 88 in the wild-type sample) and generated a high-resolution map of ssDNA distribution in this minichromosome (Fig. 3B). The ssDNA in $\triangle T X R 1$ cells were mostly clustered in the $5^{\prime}$ and $3^{\prime}$ nontranscribed sequences (NTSs) of the rDNA, with only $\sim 15 \%$ of the reads scattered in the transcribed regions (Fig. 3A,B). This is consistent with the 2D gel results showing that aberrant RIs of the rDNA 5' NTS in $\triangle T X R 1$ cells were enriched for ssDNA (Fig. 2D). Strikingly, the ssDNA distribution, represented by four distinct peaks in both forward and reverse directions, exhibited twofold rotational symmetry $(\mathrm{C} 2)$ around the replication origin ori1 (Fig. 3B). This distribution pattern fits nicely into the profile for nascent strands emanating from replication origins (Fig. 3B, schematic). Furthermore, the rDNA ssDNA distribution data indicate that ori1, but not ori2, is predominantly associated with fork stalling, maybe because the former is more active than the latter. It is worth noting that the symmetrical distribution is most likely a composite of ssDNA generation at either side of the origin in any particular DNA molecule, as simultaneous failure of both forks will be probabilistically rare. These results favor a model positing that in $\triangle T X R 1$ cells, replication elongation fails early and fails often, proximal to active replication origins. This interpretation is similar to the conclusion drawn in a recent study of replication fork stalling induced by bulky DNA alkylation agents in Saccharomyces cerevisiae (Minca and Kowalski 2011), arguing that the localized vulnerability is a general and intrinsic property of DNA replication independent of the source of replication stress.

We also separately mapped the distribution of the $5^{\prime}$ end and 3' end of the ssDNA (Fig. 3C,D). In addition to C2 symmetry, we observed distinctive fluctuations in the ssDNA distribution, with peaks coinciding with known features of the rDNA minichromosome, including ciselements involved in DNA replication (MacAlpine et al. 1997) as well as nucleosome phasing (Palen and Cech 1984). All of these may reflect the details of replication fork dynamics, especially the coordination between DNA replication and nucleosome deposition.

There were additional ssDNA peaks at the subtelomeric region (Fig. 3B-D). We also detected in $\triangle T X R 1$ cells abundant ssDNA of telomere repeats, which were not assigned to any specific genomic loci (data not shown). Taken together, these ssDNA most likely represent a dormant replication origin in the rDNA telomere, activated under the severe replication stress in $\triangle T X R 1$ cells. This result is consistent with reports that replication stress may lead to replication initiated from telomere regions in mammalian cells (Deng et al. 2009; Sfeir et al.
2009). Indeed, in certain ciliates with gene-sized chromosomes in macronuclei, DNA replication may initiate exclusively from telomeres (Skovorodkin et al. 2001). Changes in telomere homeostasis in $\triangle T X R 1$ cells, which may necessitate the telomere origin activation, were implicated by the induction of mRNA encoding telomerebinding proteins TEB1 and POT1a (Fig. 5C, below). Furthermore, we purified short nascent strands by size selection with alkaline agarose gel electrophoresis followed by BrdU immunoprecipitation and detected significant accumulation of short nascent strands at both oril and the subtelomeric region in the rDNA of $\triangle T X R 1$ cells but only at oril in wild-type cells (Fig. 3F). These results support activation of the dormant telomere origin in $\triangle T X R 1$ cells.

The rDNA regions around replication origins were significantly undercovered by Illumina sequencing relative to neighboring regions when the $\triangle T X R 1$ sample was normalized against the wild-type sample (Fig. 3E). This feature was prominent in non-rDNA chromosomes as well (Fig. 4G). Similar observations were made in deepsequencing analysis of yeast genomes (Xu et al. 2012a), which may be attributed to the loss of the small replication bubbles during sample preparation. We also performed quantitative PCR (qPCR), which yielded results largely in agreement with the Illumina sequencing data (data not shown). All of this strongly argues against localized rereplication in $\triangle T X R 1$ cells, in apparent contrast to the Arabidopsis atxr5/atxr6 mutant phenotype (Jacob et al. 2010).

\section{Genome-wide distribution of SSDNA in $\triangle T X R 1$ cells}

In $\triangle T X R 1$ cells, ssDNA distribution was also nonrandom in non-rDNA macronuclear chromosomes, revealed by a strong tendency for them to cluster (Fig. 4A; Supplemental Fig. 9A). Extrapolating from the analysis of the rDNA minichromosome, we surmised that these ssDNAenriched regions also represented replication origins in Tetrahymena. Indeed, detection of ssDNA derived from short nascent strands in cells under replication stress is a well-received methodology for mapping replication origins in various model systems (Feng et al. 2006; Gilbert 2010). Using two independently established algorithms (Qin et al. 2010; John et al. 2011), we were able to identify thousands of ssDNA clusters in $\triangle T X R 1$ cells, with significant overlap between the two data sets (Supplemental Fig. 9B-D). Here we focus on results from the HOTSPOTS algorithm, which identified 7059 ssDNA clusters with high confidence (false discovery rate $[\mathrm{FDR}]<5 \%$ ). Of note, the only two previously mapped replication origins in the non-rDNA chromosomes-ARS1-A and ARS1-B (Donti et al. 2009)—were both identified as ssDNA clusters (Fig. 4B).

Browsing through the identified ssDNA clusters, we noticed a strong tendency for them to reside in intergenic regions (Fig. 4B). This was subsequently confirmed by the composite distribution analysis (Fig. 4C-G). In the analysis, we plotted the average coverage of ssDNA in 50-bp bins, centered on the highest points of the ssDNA coverage in an identified cluster and extended in both directions for $3 \mathrm{~kb}$ (Fig. 4C). This revealed a prominent 
A

$\triangle T X R 1$ B

Random


C



D

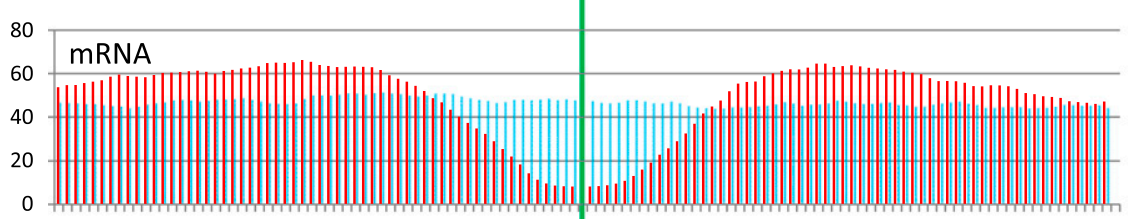

E
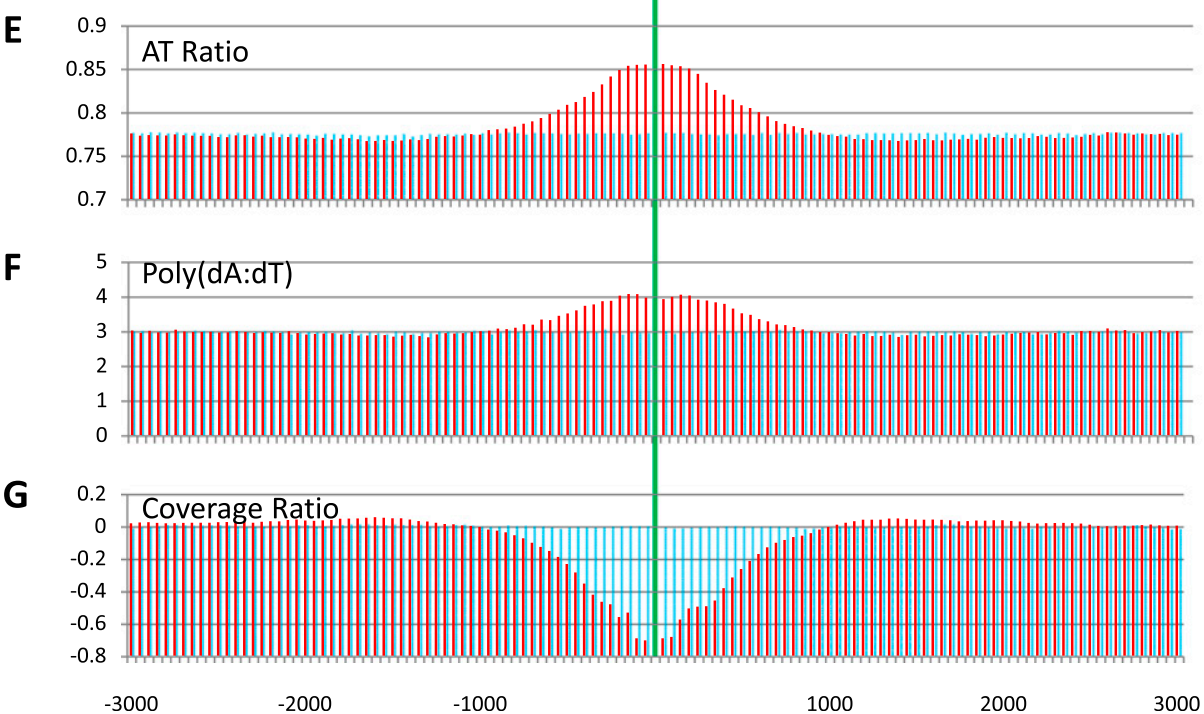

Figure 4. Global distribution of ssDNA in $\triangle T X R 1$ cells. (A) Clustering of ssDNA in $\triangle T X R 1$ cells in their distribution in non-rDNA chromosomes. The $X$-axis represents the inter-ssDNA distances (binned at 25-bp intervals and cut off at 500 bp), while the $Y$-axis represents their abundance (arbitrary units). The same total number of ssDNA were analyzed in the $\Delta T X R 1$ and randomized sample. (B) Distribution of ssDNA and mRNA in a genomic region around ARS1-A and ARS1-B, two known replication origins in non-rDNA chromosomes. (C-G) Average view analyses (binned at 50-bp intervals) of the distribution of ssDNA $(C)$, mRNA $(D)$, AT ratio $(E)$, poly$(\mathrm{dA}: \mathrm{dT})(F)$, and Illumina sequencing coverage ratio $\left(\log _{2}\right)$ of $\triangle T X R 1$ and wild-type $(\mathrm{WT})$ cells $(G)$, showing features consistent with being replication origins. The $Y$-axes represent the abundance of the studied features. The same total number of the studied features was analyzed in the $\triangle T X R 1$ and randomized sample. Details of calculation are provided in the Supplemental Material.

peak, with $>99 \%$ of the above-background ssDNA coverage in a 2-kb bracket (Fig. 4C). We performed similar analysis of mRNA coverage around the clusters based on the RNA sequencing study of Tetrahymena transcriptomes (Xiong et al. 2012). The result showed that the ssDNA clusters resided in regions depleted in mRNA transcripts but were flanked by regions enriched in transcripts (Fig. 4D). This clearly indicates that they are predominantly in intergenic regions. Base composition analysis showed that the clusters were more AT-rich (Fig. 4E), reaching the high AT ratio of intergenic regions (Eisen et al. 2006). There was also disproportional enrichment of poly(dA:dT) tracts in the ssDNA clusters (Fig. 4F), which are implicated in generating nucleosome-free regions 
characteristic of replication origins (Segal and Widom 2009). Additionally, we observed significantly reduced sequencing coverage of regions around the clusters in the $\triangle T X R 1$ sample (Fig. 4G), a feature also present in the rDNA (Fig. 3E). These features are associated with replication origins in other systems (Gilbert 2010), further strengthening the case that the ssDNA clusters in $\triangle T X R 1$ represent or include genuine replication origins in Tetrahymena. We conclude that the distribution pattern of ssDNA in $\triangle T X R 1$ cells is consistent with widespread replication stress, attributable to impaired elongation proximal to replication origins.

\section{Transcriptional responses to replication stress} in $\triangle T X R 1$ cells

Using a previously tested microarray custom-designed for Tetrahymena (Miao et al. 2009), we examined the transcriptional profile of asynchronously growing $\triangle T X R 1$ cells. We found that genes significantly induced in $\triangle T X R 1$ cells ( $\triangle T X R 1 /$ wild type $>2$ ) were concentrated in a tight cluster of functionally related genes delineated by gene ontology (GO) analysis (Fig. 5A; Supplemental Fig. 10A), with the most prominently affected categories including the response to DNA damage stimulus, DNA repair, and the DNA metabolic process (Fig. 5B). Accordingly, we assembled a list of genes distributed in the highly conserved pathways and presented the phylogenetic analysis along with the microarray data (Fig. $5 \mathrm{C}$ ). The list includes key players in DNA repair and DNA replication in addition to the ssDNA-binding and ssDNA-sensing pathways mentioned earlier. There is also a large array of DNA helicases, many of which are specific for branched structures generated during DNA replication and DNA repair. These conserved genes represent the core group of the transcriptional response in $\triangle T X R 1$ cells, which were validated by qPCR analysis (Fig. 5D; Supplemental Fig. 10B; Supplemental Table 1). Some of the most highly induced genes are in the base excision repair (BER) pathway, including Tetrahymena homologs to human PARP1, 3-alkyladenine-DNA glycosylases, and bacterial AlkD DNA glycosylases. This is probably attributable to the abundance of ssDNA, which is vulnerable to alkylation damages (Friedberg et al. 2006). We also noted induction of genes needed for HR, including MRE11, ATR1, APO1, EXO1, DNA2, RPA1, RAD51, RAD5, RAD54, SGS1, TOP3A, RMI1, MUS81, EME1, MND1, HOP2B, and FANCM. They are potentially involved in various steps of $\mathrm{HR}$, from end resection to Holliday junction resolution (San Filippo et al. 2008). DNA polymerases, especially those involved in error-prone translesion synthesis, were also induced, probably for filling in of ssDNA regions (Malkova and Haber 2012). The majority of these genes are also highly induced in critical stages of sexual reproduction (Supplemental Fig. 10C), a coordination potentially reflecting the presence of a common transcription activation pathway analogous, if not homologous, to the ATR-mediated transcriptional response in S. cerevisiae (Polo and Jackson 2011).

We also performed microarray analysis of HU-treated wild-type cells, which showed a wide spectrum of induced genes, including many involved in general metabolism (Supplemental Fig. 10A). Nonetheless, there was substantial overlap with the core group of the transcriptional response in $\triangle T X R 1$ cells (Fig. 5B,C). Indeed, in comparison with HU-treated cells, the transcriptional response in $\triangle T X R 1$ cells was much more focused on DNA replication/ DNA repair and significantly stronger (Fig. 5C; Supplemental Fig. 10A). These results corroborate the presence of replication stress in $\triangle T X R 1$ cells, which is attributable to defects in replication elongation. Intriguingly, TXR1 was highly induced by HU treatment (data not shown), supporting that it plays some roles in stabilizing stressed replication forks (consistent with its deletion causing replication stress).

As a control, we analyzed $\triangle E Z L 2$ cells, which deleted a Tetrahymena homolog of Drosophila E(z), the canonical Polycomb group protein involved in H3K27 methylation, heterochromatin formation, and transcriptional repression (Grossniklaus and Paro 2007). Even though a diverse group of genes was induced in $\triangle E Z L 2$ cells, there was little overlap with the core group of the transcriptional response in $\triangle T X R 1$ cells (Fig. 5C,D; Supplemental Fig. 10A), supporting the functional separation of TXR1 and EZL2.

\section{Deficiency in H3K27me1 underlying replication stress in $\triangle T X R 1$ cells}

Relative to their counterparts in wild-type and $\triangle E Z L 2$ cells, macronuclei of $\triangle T X R 1$ cells were larger in size, punctuated with regions poorly stained with DAPI (Fig. 6A). Interestingly, many of the ssDNA foci in G2 and amitotic macronuclei coincided with these regions (Fig. 1A), probably representing decondensed chromatin in which many transactions involving DNA, including post-replication repair, occur (Solimando et al. 2009). Macronuclei of $\triangle T X R 1$ cells were also much more fragile, as evidenced by increased sensitivity to detergent extraction (data not shown). In addition, a significant amount of chromatin was detected as DAPI-stained fine filaments at the periphery of macronuclei, forming a halo-like structure (Fig. 6A). These characteristics are consistent with chromatin decondensation, as reported in the Arabidopsis atxr5/atxr6 hypomorphic mutant (Jacob et al. 2009, 2010). We also observed dramatic changes in the organization of nucleoli (Fig. 6B). In wild-type growing cells, nucleoli are evenly distributed as fine granules at the periphery of macronuclei (Gorovsky 1973). In strong contrast, nucleoli were aggregated in $\triangle T X R 1$ growing cells, as detected by fluorescence in situ hybridization (FISH) using an rDNA probe (Fig. 6B). All of these results support the presence of both global and local chromatin defects in $\triangle T X R 1$ cells.

We systematically analyzed PTMs of all four core histones in logarithmically growing wild-type, $\triangle T X R 1$, and $\triangle E Z L 2$ cells by quantitative MS using ${ }^{15} \mathrm{~N}$-labeled histones as the internal standard (Fig. 6C,D; Supplemental Fig. 11). Our results revealed in $\triangle T X R 1$ cells a dramatic reduction of H3K27me1 levels as well as progressively diminished effects on H3K27me2 and H3K27me3 (Fig. 6C,D). The result is consistent with the moderate reduction of $\mathrm{H} 3 \mathrm{~K} 27 \mathrm{mel}$ levels 




B
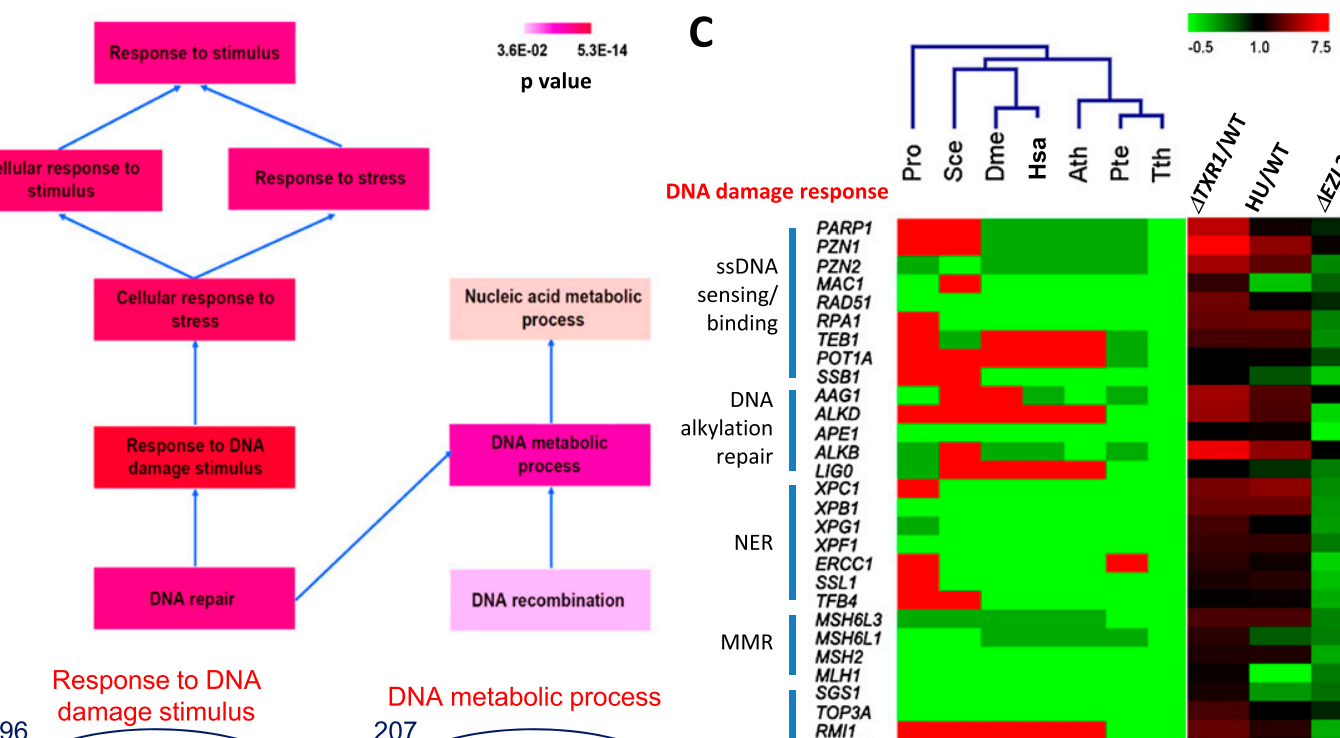

DNA damage response
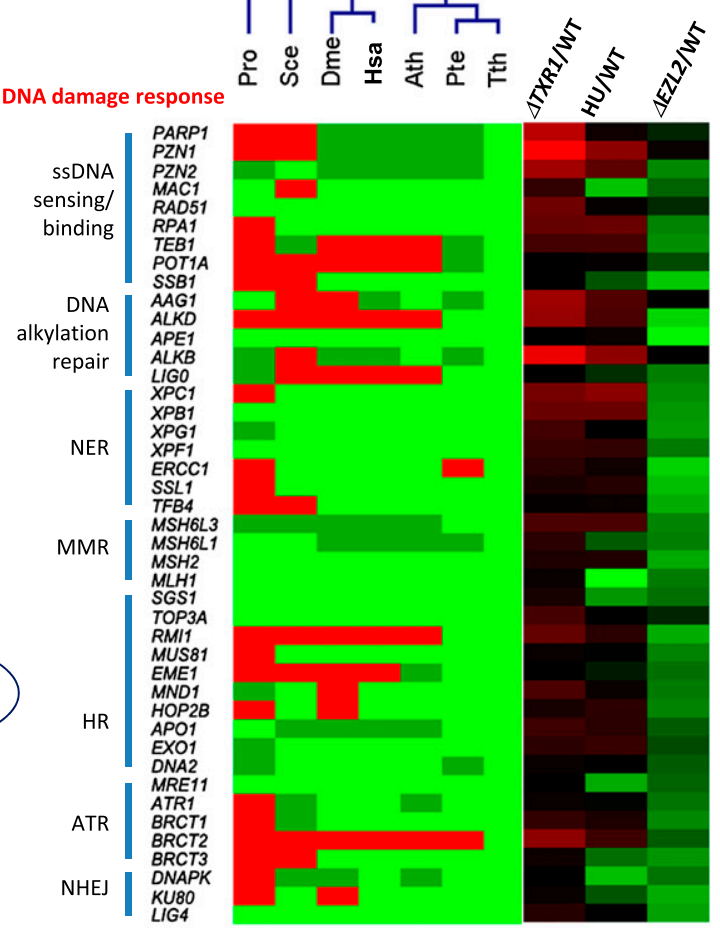

D
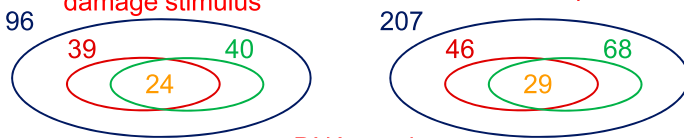

DNA repair
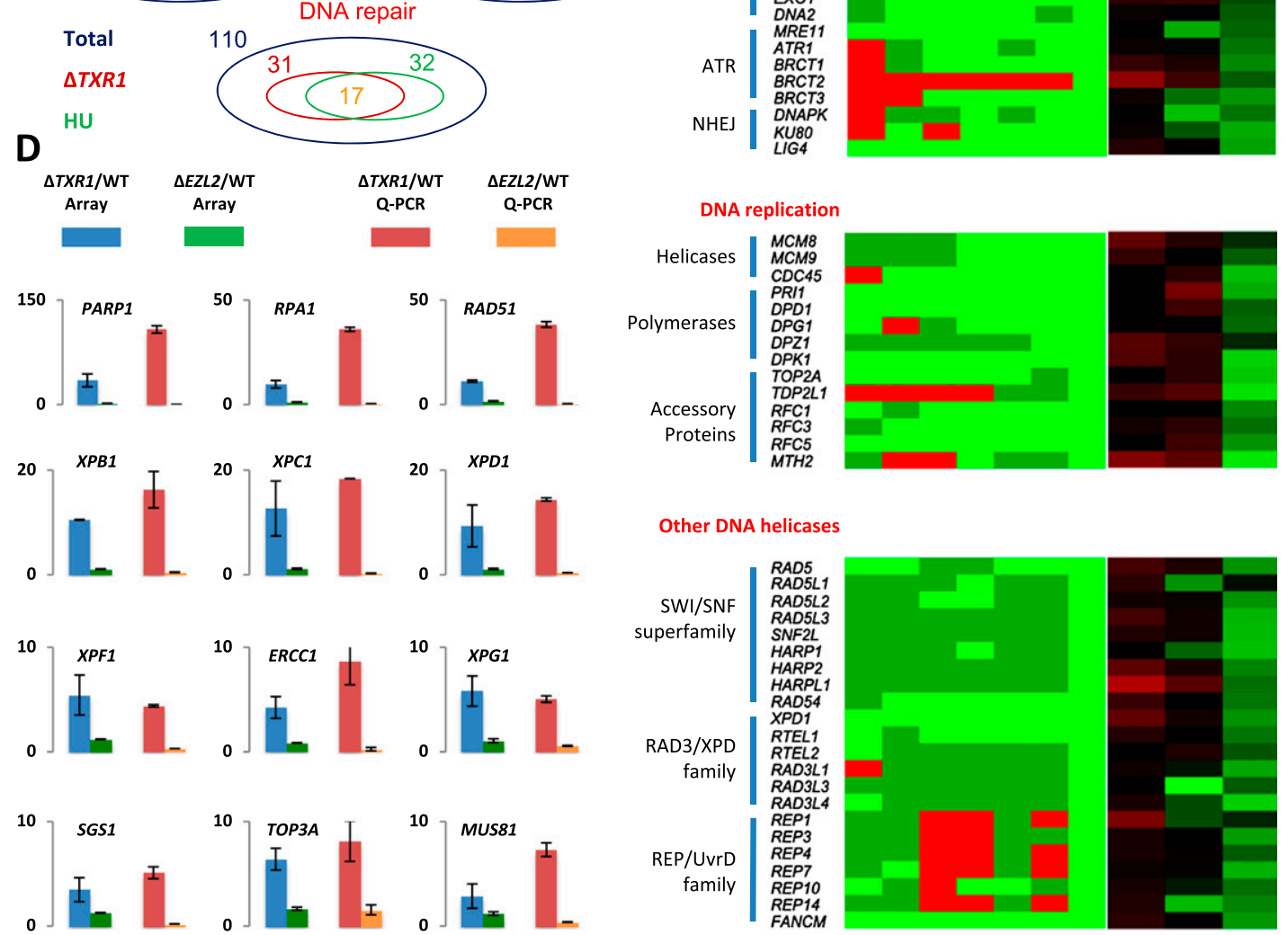

Figure 5. Transcriptional responses to replication stress in $\triangle T X R 1$ cells. $(A)$ Induction of a cluster of functionally related genes in $\triangle T X R 1$ cells, revealed by GO analysis (GO term: biological process) and colored according to the $P$-value. $(B)$ Induction of many genes in the categories of response to DNA damage, DNA repair, and DNA metabolic process in $\triangle T X R 1$ and HU-treated cells, with significant overlap between them. $(C)$ The core of transcriptional responses in $\triangle T X R 1$ cells, as revealed by phylogenetic and microarray analysis, is similar to those in HU-treated cells but not $\triangle E Z L 2$ cells. The left panels are colored as the following: (green) homologs identified by the best hits of BLASTP search in both directions; (dark green) homologs identified by the best hits in one direction; (red) no homolog. (Pro) An assembly of prokaryotes (see the Supplemental Material); (Sce) S. cerevisiae; (Dme) Drosophila melanogaster; (Hsa) Homo sapiens; (Ath) Arabidopsis thaliana; (Pte) Paramecium tetraurelia; (Tth) T. thermophila. The right panels are colored according to the ratio ( $\left.\log _{2}\right)$ of transcript levels of the mutants or treated cells normalized against the wild-type (WT) controls. (NER) Nucleotide excision repair; (MMR) mismatch repair; (HR) homologous recombination; (NHEJ) nonhomologous end joining. $(D)$ Representative genes highly induced in $\triangle T X R 1$ but not $\triangle E Z L 2$ cells, shown by both microarray and qPCR analysis. 
A
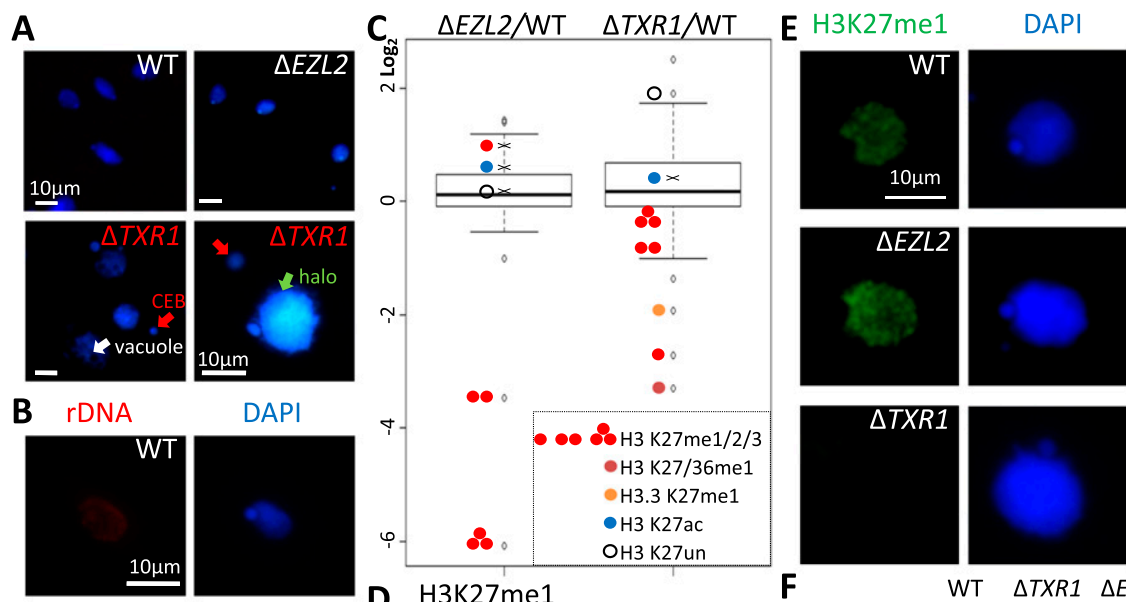

DAPI
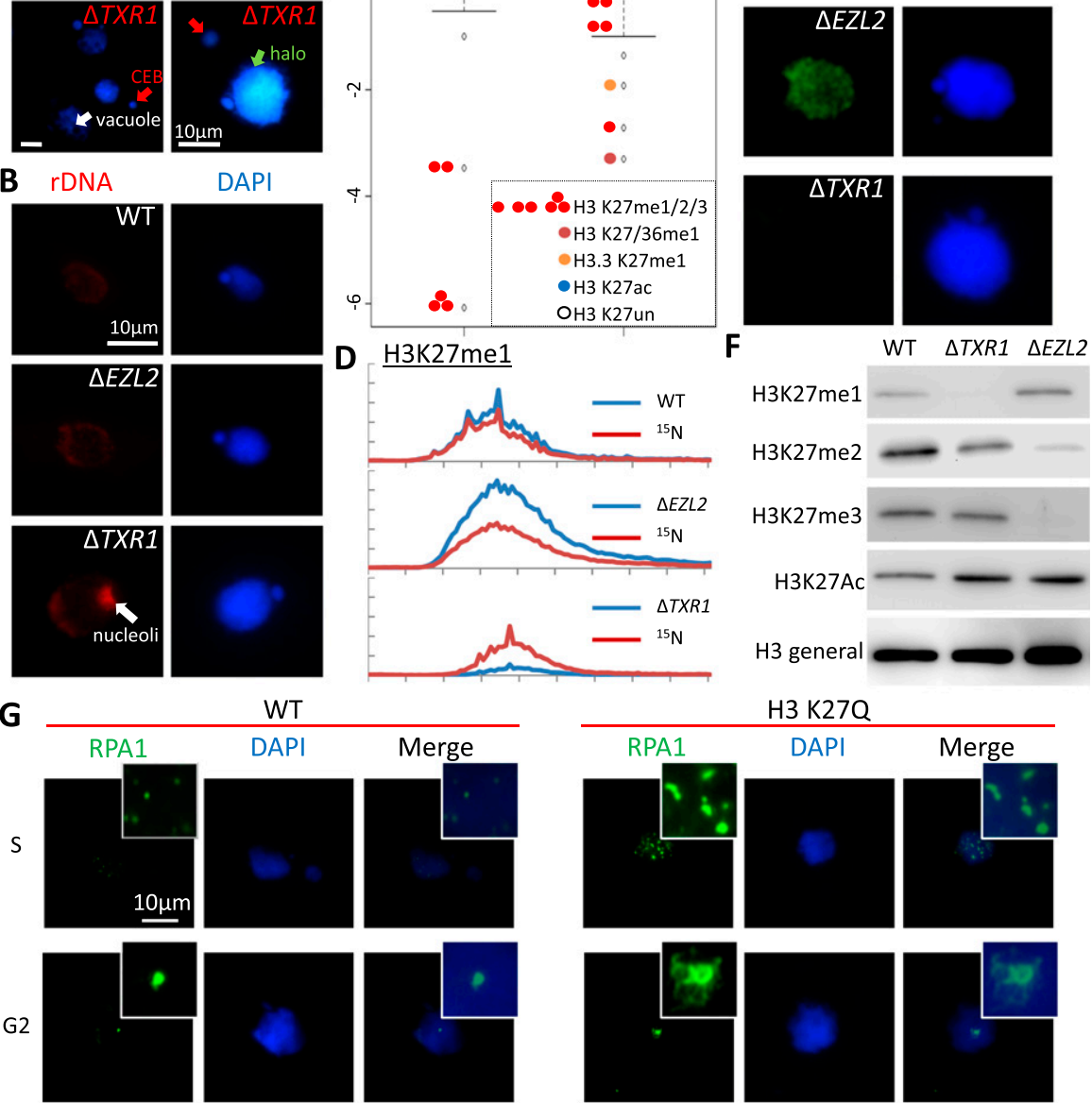

H3 K27Q
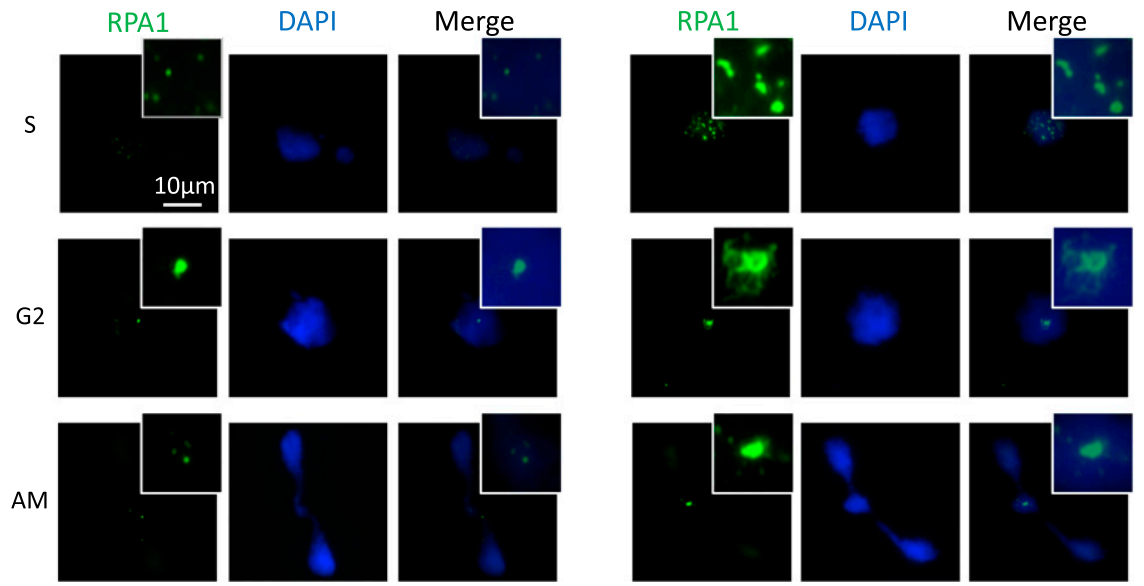

Figure 6. Replication stress in $\triangle T X R 1$ cells is mediated by a deficiency in H3K27me1. (A) Abnormal nuclear morphology of $\Delta T X R 1$ cells, revealed by DAPI staining. (B) Abnormal aggregation of nucleoli in $\triangle T X R 1$ cells, revealed by rDNA FISH (probe corresponding to the $5^{\prime}$ NTS). (White arrow) Aggregated nucleoli at the periphery of the macronucleus. $(C)$ Box plot analysis of PTMs in all four core histones quantified by MS, which covers 53 combinations of PTMs and their unmodified counterparts (see Supplemental Fig. 11 for a complete list). All outliers in the mutant/wild-type (WT) ratio are marked with open ovals. H3K27-related species are denoted with symbols specified in the inset. $(D)$ MS quantification of H3K27me1 levels in wild-type, $\triangle T X R 1$, and $\Delta E Z L 2$ cells. ${ }^{15} \mathrm{~N}$-labeled histone H3 was used as the internal standard. (E) H3K27me1 deficiency in $\triangle T X R 1$ cells, shown by immunofluorescence staining with the modification-specific antibody. (F) H3K27 methylation deficiency in $\triangle T X R 1$ and $\triangle E Z L 2$ cells, shown by immunoblotting with the modification-specific antibodies. $(G)$ Accumulation of RPA1 in H3 K27Q cells. Note its similarity to $\Delta T X R 1$ and HU-treated cells.

observed in the Arabidopsis atxr5/atxr6 hypomorphic mutant (Jacob et al. 2009). In $\triangle E Z L 2$ cells, there was little H3K27me2 or H3K27me3 (Fig. 6C), as expected of an E(z) family HMT (Grossniklaus and Paro 2007), while H3K27me1 accumulated (Fig. 6C,D). H3K27 acetylation (H3K27ac) levels remained relatively stable in $\triangle T X R 1$ and $\triangle E Z L 2$ cells (Fig. 6C,F; Supplemental Fig. 11). These findings were corroborated by immunofluorescence staining and immunoblotting with modification-specific antibodies (Fig. 6E,F). The results support that TXR1 is responsible for the bulk of $\mathrm{H} 3 \mathrm{~K} 27 \mathrm{mel}$, while EZL2 is mainly required for $\mathrm{H} 3 \mathrm{~K} 27 \mathrm{me} 2$ and $\mathrm{H} 3 \mathrm{~K} 27 \mathrm{me} 3$, as they jointly control the three states of H3K27 methylation in macronuclei of growing Tetrahymena cells. Neither replication stress nor abnormal nuclear morphology was observed in $\triangle E Z L 2$ cells (Fig. 6A,B; data not shown), 
emphasizing distinct roles played by TXR1-dependent $\mathrm{H} 3 \mathrm{~K} 27 \mathrm{mel}$ in DNA replication.

We next addressed whether the function of TXR1 was dependent on the HMT activity conferred by its SET domain. Indeed, we detected strong BrdU signals, indicative of ssDNA accumulation, in the catalytically dead mutant of TXR1 (Y526N), phenocopying $\triangle T X R 1$ (Fig. 7C). The result rules out the possibility that TXR 1 mainly functions as a structural platform. After establishing the importance of the HMT activity of TXR1, we further addressed whether H3K27 was its functionally relevant substrate. Taking advantage of a previously established system for histone mutagenesis (Liu et al. 2004), we generated strains in which the only expressed major $\mathrm{H} 3$, $\mathrm{H} 3.2$, carries the K27Q mutation. In H3.2 K27Q cells, we detected elevated levels of RPA1 as a sensitive indicator of ssDNA accumulation and replication stress (Fig. 6G). We can directly attribute the replication phenotype in H3.2 K27Q cells to blocking of monomethylation at this residue, as we just ruled out potential roles of other PTMs in DNA replication.

\section{Interaction with PCNA involved in targeting TXR1 to elongating replication forks}

TXR1 also features a putative PIP box (Supplemental Fig. 12A) by which proteins are recruited to sites of active DNA replication and DNA repair (Maga and Hubscher 2003). To validate that the sequence motif is a bona fide PIP box, we performed peptide pull-down assays with the TXR1 PIP peptide (residues 207-219, TDQKLIEDYFIRG). Using the Tetrahymena whole-cell extract as well as the recombinant protein as the input, we were able to specifically enrich Tetrahymena PCNA in the pull-down (Fig. 7A). The binding was competed away by the TXR1 PIP peptide but not by a similar peptide with mutations in the conserved aromatic residues (Y215A F216A) (Fig. 7B). We also generated Tetrahymena strains with the same PIP mutations. The derived mutant (Y215A F216A) showed DNA replication defects phenocopying $\triangle T X R 1$, while strains transformed with the wild-type construct were normal (Fig. 7C). This is consistent with functional analyses of PIP in Arabidopsis ATXR5 and ATXR6 (Raynaud et al. 2006; Jacob et al. 2010). PCNA distribution, revealed by immunofluorescence staining, was not affected in any of the TXR1 loss-of-function mutants (Fig. 7C), suggesting that PCNA is upstream of TXR1 in the pathway. Taken together, these results support that PCNA plays a conserved role in recruiting this subfamily of $\mathrm{H} 3 \mathrm{~K} 27 \mathrm{me}$ specific HMTs to sites of replication elongation.

\section{Discussion}

\section{Regulation of replication elongation by TXR1}

As duplication of genetic information is coordinated with duplication of epigenetic information, perturbations to one can conceivably affect the other. In this study, we demonstrated that in Tetrahymena cells lacking TXR1, encoding a conserved HMT, there is severe replication stress. First, there is accumulation of RIs. Slowing and stalling of replication fork progression can increase the levels of normal RIs and reduce their turnover rates, as shown by both alkaline and neutral agarose gel electrophoresis. There



Figure 7. A TXR1-dependent, H3K27me1-mediated pathway regulating replication elongation. (A) TXR1 PIP peptide pull-down of PCNA, with recombinant PCNA or cell lysate as the input. (B) Effective competition by the PIP peptide (50-200 $\mu M)$ but not a similar peptide with mutations in the conserved aromatic residues (PIP-AA). (C) ssDNA and PCNA distribution in TXR1 mutants, including the PIP (Y215A F216A) and SET (Y526N) mutant. (D) Model for origin-proximal replication elongation defects in $\Delta T X R 1$ cells. $(E)$ Model for a TXR1-dependent, H3K27me1-mediated pathway regulating replication elongation. 
is also strong support for the accumulation of DSB as abnormal RIs. Importantly, we demonstrated fork stalling as well as formation of recombination intermediates for restarting stalled forks with the $2 \mathrm{D}$ gel. It is worth noting that accumulation of recombination intermediates prevents sister chromatid separation; thus, difficulties in DNA replication can potentially lead to difficulties in DNA distribution later in the cell cycle. A possible manifestation is ssDNA-enriched regions in the G2 and dividing macronuclei in $\triangle T X R 1$ cells. In Tetrahymena, the entangled DNA is disposed through CEBs, hinting at similar functions for the micronuclei, an analogous structure in mammalian cells (Fenech et al. 2011). Second, there is accumulation of ssDNA. We visualized ssDNA in multiple ways, including direct detection of incorporated BrdU exposed under nondenaturing conditions and indirect detection through the ssDNA-binding protein RPA1 and the ssDNA-sensing signal $\gamma$-H2A.X. From the 2D gel data, we inferred the association of ssDNA with stalled and/or broken replication forks. We also used a novel analysis of the paired-end Illumina sequencing data to identify ssDNA. The genome-wide distribution pattern of ssDNA strongly suggests that they are enriched around replication origins. Third, there are robust DDRs. These include activation of the conserved ATR and PARP signaling pathways. There are also transcriptional responses typically induced by replication stress. These induced genes may be involved in stabilizing stressed replication forks by countering the formation of detrimental RIs and in restarting stalled replication forks by HR and other DNA repair processes. Prominent among them are Tetrahymena homologs to XPF/ERCC1, $X P G, M U S 81, E X O 1$, and DNA2, which are structurespecific nucleases that can target replication and recombination intermediates (Schwartz and Heyer 2011) as well as structure-specific DNA helicases (Yusufzai and Kadonaga 2011), including Tetrahymena homologs to $X P B, X P D$, BLM, RAD54, RAD5, HARP, FANCM, and UVrD/REP.

We favor the model that replication stress in $\triangle T X R 1$ cells is caused by defects in replication elongation. In addition to the aforementioned results, this is also supported by the similarity in the phenotype of $\triangle T X R 1$ and HU-treated cells. In addition, $\triangle T X R 1$ was phenocopied by the TXR1 PIP mutant, functionally linking TXR1 with PCNA, a DNA polymerase processivity factor (Moldovan et al. 2007). Our data do not corroborate the alternative rereplication model proposed for the Arabidopsis atxr5/ atxr6 hypomorphic mutant (Jacob et al. 2010). Indeed, accumulation of copy number variants in the Arabidopsis mutant may be an indirect consequence of replication stress, as observed in human cells treated with aphidicolin or HU (Arlt et al. 2012).

Furthermore, we propose that replication elongation is specifically challenged around replication origins in $\triangle T X R 1$ cells (Fig. 7D). This is mainly based on the distribution pattern of ssDNA. First, abundant ssDNA are mapped proximal to the rDNA oril at nucleotide resolution, revealing four peaks with C2 symmetry characteristic of nascent strands emanating from replication origins. Second, abundant ssDNA are mapped to rDNA subtelomeric regions, coinciding with activation of a dormant origin in rDNA telomere. Third, ssDNA clusters are found in genomic regions containing ARS1-A and ARS1-B, the only two functionally characterized replication origins in non-rDNA chromosomes. Fourth, ssDNA clusters are highly enriched in intergenic regions, with features characteristic of replication origins in other lower eukaryotic systems. The scenario in $\triangle T X R 1$ cells is strikingly similar to replication fork stalling around origins caused by bulky DNA alkylation agents in S. cerevisiae (Minca and Kowalski 2011). This strongly suggests that emerging replication forks emanating from proximal origins behave differently from the more established replication forks (Fig. 7D). They are prone to stalling due to either insufficiency in composition or conformation of the assembled replisome (compared with mature forks) or lack of stabilizing effects provided by the DDR (compared with HR-competent forks). Importantly, all of this also implicates replication origins as hot spots for replication stressinduced mutations. The mechanism underlying the vulnerability of emerging replication forks as well as the roles played by TXR1 in their stabilization need to be further explored.

The origin-proximal elongation stress model that we present here is a parsimonious synthesis of compatible hypotheses supported by the collective data. Here we address alternative interpretations for some results and explain our considerations for the overall framework. First, elongation defects in $\triangle T X R 1$ cells may activate dormant origins as an alternative (but not a mutually exclusive one) to HR for restarting replication. This is supported by activation of the rDNA telomeric origin. In addition, the apex of the $\mathrm{X}$ spike in the $2 \mathrm{D}$ gel can also be interpreted as converging replication forks emanating from origins in both halves of the rDNA palindrome. A related RI pattern is detected during rDNA amplification due to the presence of a developmentally programmed replication fork barrier near the palindromic center (Zhang et al. 1997). Second, there is a potential cause and effect problem concerning replication stress and changes in transcription. We conclude that the transcription response is the result of replication stress in $\triangle T X R 1$ cells rather than the cause of replication stress. This is strongly supported by induction of genes that can alleviate rather than aggravate replication stress. In addition, H3K27me1 is a highly abundant ( $>30 \%$ occurrence estimated by MS) and presumably widely distributed mark; therefore, it is unlikely to be specifically involved in transcription regulation of a narrow array of target genes and more likely to be involved in a genome-wide process like replication. Last, we are aware that we have only circumstantial evidence for all but a few replication origins in non-rDNA chromosomes, even though we argue that the ssDNA clusters that we identified should represent or include them. This can be corroborated by independent mapping of genome-wide distribution of the ORC or CMG complex components in future studies. Our discovery that sites sensitive to elongation stress either map to known origins or have the hallmarks of origins leads us to speculate that the transition from replication initiation 
to elongation is more intimately coordinated than previously thought, a process in which epigenetic regulation plays a prominent role.

\section{Potential roles for H3K27me1 and other lysine monomethylation events in DNA replication}

Comprehensive analyses of histone modifications in $\triangle T X R 1$ and $\triangle E Z L 2$ cells have revealed that reduced levels of H3K27me1 are strongly correlated with replication defects as well as the abnormal chromatin structure and nuclear morphology. It remains to be elucidated whether chromatin decondensation is the direct result of reduced levels of $\mathrm{H} 3 \mathrm{~K} 27 \mathrm{me} 1$ or an indirect consequence of replication defects. Nonetheless, there is a clear division of labor between TXR1-catalyzed H3K27me1 and EZL2catalyzed H3K27me2/H3K27me3 in replication and transcription, respectively. In addition, we observed ssDNA accumulation and replication stress in $\mathrm{H} 3 \mathrm{~K} 27 \mathrm{Q}$ cells, strengthening the DNA replication role of $\mathrm{H} 3 \mathrm{~K} 27 \mathrm{mel}$. The relatively mild replication phenotype in the H3 mutant may be attributable to the presence of quantitatively minor $\mathrm{H} 3$ variants H3.3 and H3.4 (Yu and Gorovsky 1997; Cui et al. 2006). As H3.3 was also monomethylated at Lys 27 (Zhang et al. 2013), it can potentially compensate the major $\mathrm{H} 3$ function. Histone mutagenesis studies addressing the $\mathrm{H} 3$ variants can further clarify the roles played by H3K27mel in DNA replication.

Based on all the evidence, we propose the presence of a TXR1-dependent, H3K27me1-mediated pathway that regulates replication elongation. In particular, we posit a loop model for the pathway, in which TXR1 and H3K27me1 are pivotal links coupling histone modifications with DNA replication (Fig. 7E). The TXR1 pathway intersects the DNA replication pathway in the upstream, as PCNA plays an important role in recruiting TXR1. For the replication defects to manifest, additional intersections must also occur downstream. H3K27me1 may affect the dynamics of nucleosome disassembly and reassembly before and after replication forks, which may or may not be mediated by histone chaperones. Alternatively, H3K27me1 may modulate the affinity of histone-binding effectors. These effectors can be components of the replisome or the DDR pathway, which help to stabilize emerging replication forks. There is also a possibility for TXR1 to directly methylate and modulate nonhistone substrates.

The TXR1 subfamily of HMTs is conserved in protozoa and plants (Supplemental Fig. 12B,C). Even though there are no TXR1 homologs in metazoa, the function of H3K27me1 in DNA replication may still be conserved. In Tetrahymena and plants, H3K27mel is deposited by TXR1 and its homologs, while $E(z)$ homologs builds up H3K27me2 and H3K27me3 levels (Jacob et al. 2009; Zhang et al. 2013). In metazoa, all three states of H3K27 methylation are deposited by $E(z)$ homologs, which are also associated with regions of active DNA replication (Hansen et al. 2008; Shen et al. 2008; Petruk et al. 2012). Furthermore, H3K27me1 levels of newly synthesized H3 are elevated specifically at $S$ phase in mammalian cells $(\mathrm{Xu}$ et al. 2012b), implicating a role in replication. Intriguingly, other lysine monomethylation events can also affect replication in metazoa. SetDB1 deposits H3K9mel and is associated with replication-coupled histone deposition, especially in heterochromatin regions (Loyola et al. 2006, 2009). SETD8/PR-Set7, specific for H4K20me1, is recruited by PCNA to directly function at the replication fork and is essential for S-phase progression (Beck et al. 2012). G9a/ KMT1C deposits H3K56me1, which regulates replication through its interaction with PCNA (Yu et al. 2012). All of this suggests deep mechanistic connections between lysine monomethylation and DNA replication to be elucidated in the future.

\section{Materials and methods}

Additional details on all of the methods are available in the Supplemental Material.

\section{Strains and culture conditions}

Tetrahymena wild-type strains CU427 and CU428 (Tetrahymena Stock Center) and mutant strains (Supplemental Table S2) were grown in the SPP medium (Sweet and Allis 1998). $\triangle T X R 1$ was a homozygous homokaryon strain generated by genetic manipulation of germline transformants (Hai and Gorovsky 1997), with paromomycin resistance conferred by a neo4 cassette (Mochizuki 2008). To introduce HA-tagged RPA1, PARP1, and PCNA into $\triangle T X R 1$ cells, a newly developed cassette conferring cycloheximide resistance $(\operatorname{ch} x)$ was used as the selection marker.

\section{Fluorescence staining and imaging}

For immunofluorescence staining, cells were fixed in $2 \%$ paraformaldehyde (PBS) and permeabilized with $0.4 \%$ Triton X-100 (PBS) before incubation with the appropriate antibodies (Liu et al. 2007). TUNEL assay was performed with Click-iT TUNEL Alexa Fluor imaging assay kit (Invitrogen). FISH was performed as described (Mochizuki et al. 2008), with modifications. Digital images were collected using a Carl Zeiss Axio Imager Z1 microscope with a Carl Zeiss AxioCam HRc camera.

\section{Alkaline and neutral agarose gel electrophoresis}

Sample plugs were prepared as described (Cassidy-Hanley et al. 2005). Gel electrophoresis was performed in the neutral (1X TAE) or alkaline buffer (0.1 M NaOH, $2 \mathrm{mM}$ EDTA at $\mathrm{pH} \geq 13.2)$ overnight. DNA was either alkaline-transferred $(0.4 \mathrm{mM} \mathrm{NaOH}$ for $2 \mathrm{~h}$ ) to Nylon $\mathrm{N}^{+}$membrane (GE Healthcare) or in-gel-trapped (Khan and Nawaz 2007) before being probed by $\alpha$-BrdU antibody.

\section{$2 D$ neutral-neutral agarose gel electrophoresis}

2D gel was performed as described (MacAlpine et al. 1997). Briefly, purified genomic DNA was digested with HindIII, and RIs were enriched by BND cellulose (Sigma) where indicated.

\section{Illumina sequencing and data analysis}

Illumina sequencing libraries were constructed according to manufacturer-recommended protocols. Filtered reads were mapped to the T. thermophila macronuclear genome using the TopHat-Fusion program (Kim and Salzberg 2011). These data were used to identify inverted repeats representing ssDNA in the $\triangle T X R 1$ and wild-type samples. The SAMtools program (Li et al. 
2009) and custom scripts were used to calculate the sequencing coverage of each position across the genome.

\section{ssDNA cluster identification}

The HOTSPOT program (John et al. 2011) was used to identify ssDNA clusters in all scaffolds $>10 \mathrm{~kb}$. As a control, ssDNA distribution was randomized, and ssDNA clusters in it were also identified with HOTSPOT. FDR was calculated by comparing the real and random data (John et al. 2011). The HPeak program (Qin et al. 2010) was also used to identify ssDNA clusters in all scaffolds >10 kb (http://www.sph.umich.edu/csg/qin/HPeak).

\section{Microarray and $q P C R$ analysis}

Microarray experiments were performed to investigate global gene expression changes in $\triangle T X R 1, \triangle E Z L 2$, and HU-treated wild-type cells using the Roche NimbleGen platform (Gene Expression Omnibus [GEO] accession GPL6759) (Miao et al. 2009). qPCR validation of gene expression levels was performed with randomly primed cDNA as the template and gene-specific primers (Supplemental Table S3).

\section{Analysis of differentially expressed genes}

GO enrichment analysis (Ashburner et al. 2000) was performed to investigate the significantly induced function categories (GO terms). FDR was used for multiple testing correction, and only function categories significantly overrepresented $(P<0.05)$ were plotted and subjected to further analysis. A group of 79 genes with highly correlated functional annotations and GO terms were selected as the core group of transcriptional responses in $\triangle T X R 1$ cells. The expression cluster analysis and the heat map were generated in the $\mathrm{MeV}$ program (version 4.6.2) (Mar et al. 2011).

To investigate the conservation of these 79 genes, all-against-all BLASTP analysis (Altschul et al. 1997) was performed between T. thermophila and other model eukaryotes or prokaryotes. All prokaryotic species were combined as one entity in a homology search. Reciprocal BLASTP best hits were interpreted as orthologs, and single-directional BLASTP best hits were interpreted as homologs. MeV software (Mar et al. 2011) was used for heat map construction and cluster analysis.

\section{Quantitative liquid chromatography (LC)-MS analysis} of histone PTMs

MS-based relative quantification of PTMs in all four core histones was performed using ${ }^{15} \mathrm{~N}$-labeled histones from wildtype Tetrahymena cells as internal standards for all LC-MS runs, as described (Zhang et al. 2013).

\section{Peptide pull-down}

Peptide pull-down assays were performed using whole-cell lysate or purified recombinant PNCA protein as described (Wysocka 2006), with modifications. Biotinylated PIP peptide of TXR1 (TDQKLIEDYFIRGGK-biotin) was bound to avidin agarose beads (Pierce). Avidin beads without prebound PIP peptide were included as the negative control.

\section{Data deposition}

The microarray data were deposited into the National Center for Biotechnology Information (NCBI) GEO database with the accession number GSE44990. The deep-sequencing data were deposited into the Sequence Read Archive (SRA) database with the accession number SRA079862.

\section{Acknowledgments}

We thank Martin A. Gorovsky, Thomas E. Wilson, and Xiaochun Yu for critical reading of the manuscript. We thank Xiaowen Zou and Houjun He (Genergy Biotechnology Co., Ltd., Shanghai) for their help in Illumina sequencing. This work was supported by NIH R01-GM087343 to Y.L., National Natural Science Foundation of China (31071993) and Fund of State Key Laboratory of Genetics Resources and Evolution (GREKF10-09) to W.M., NIH 1P41RR018627 to P.C.A., NIH R01-GM53572 to G.M.K., NIH R01-HG005119 to Z.Q., and University of Michigan Rackham Graduate Student Research Grant to C.Z.

\section{References}

Aguilera A, Gomez-Gonzalez B. 2008. Genome instability: A mechanistic view of its causes and consequences. Nat Rev Genet 9: 204-217.

Altschul SF, Madden TL, Schaffer AA, Zhang JH, Zhang Z, Miller W, Lipman DJ. 1997. Gapped BLAST and PSI-BLAST: A new generation of protein database search programs. Nucleic Acids Res 25: 3389-3402.

Amin AA, Pearlman RE. 1985. Autonomously replicating sequences from the non transcribed spacers of Tetrahymena thermophila ribosomal DNA. Nucleic Acids Res 13: 26472659.

Arlt MF, Wilson TE, Glover TW. 2012. Replication stress and mechanisms of CNV formation. Curr Opin Genet Dev 22: 204-210.

Ashburner M, Ball CA, Blake JA, Botstein D, Butler H, Cherry JM, Davis AP, Dolinski K, Dwight SS, Eppig JT, et al. 2000. Gene ontology: Tool for the unification of biology. Nat Genet 25: 25-29.

Avvakumov N, Nourani A, Cote J. 2011. Histone chaperones: Modulators of chromatin marks. Mol Cell 41: 502-514.

Beck DB, Oda H, Shen SS, Reinberg D. 2012. PR-Set7 and H4K20me1: At the crossroads of genome integrity, cell cycle, chromosome condensation, and transcription. Genes Dev 26: 325-337.

Branzei D, Foiani M. 2010. Maintaining genome stability at the replication fork. Nat Rev Mol Cell Biol 11: 208-219.

Broderick S, Rehmet K, Concannon C, Nasheuer HP. 2010. Eukaryotic single-stranded DNA binding proteins: Central factors in genome stability. Subcell Biochem 50: 143-163.

Cassidy-Hanley D, Bisharyan Y, Fridman V, Gerber I, Lin C, Orias E, Orias JD, Ryder H, Vong L, Hamilton EP. 2005. Genome-wide characterization of Tetrahymena thermophila chromosome breakage sites. II. physical and genetic mapping. Genetics 170: 1623-1631.

Cech TR, Brehm SL. 1981. Replication of the extrachromosomal ribosomal RNA genes of Tetrahymena thermophilia. Nucleic Acids Res 9: 3531-3543.

Coyne RS, Thiagarajan M, Jones KM, Wortman JR, Tallon LJ, Haas BJ, Cassidy-Hanley DM, Wiley EA, Smith JJ, Collins K, et al. 2008. Refined annotation and assembly of the Tetrahymena thermophila genome sequence through EST analysis, comparative genomic hybridization, and targeted gap closure. BMC Genomics 9: 562.

Cui B, Liu Y, Gorovsky MA. 2006. Deposition and function of histone $\mathrm{H} 3$ variants in Tetrahymena thermophila. Mol Cell Biol 26: 7719-7730.

Deng Z, Norseen J, Wiedmer A, Riethman H, Lieberman PM. 2009. TERRA RNA binding to TRF2 facilitates heterochromatin 
formation and ORC recruitment at telomeres. Mol Cell 35: 403-413.

Doksani Y, Bermejo R, Fiorani S, Haber JE, Foiani M. 2009. Replicon dynamics, dormant origin firing, and terminal fork integrity after double-strand break formation. Cell 137: 247258.

Donti TR, Datta S, Sandoval PY, Kapler GM. 2009. Differential targeting of Tetrahymena ORC to ribosomal DNA and nonrDNA replication origins. EMBO I 28: 223-233.

Eisen JA, Coyne RS, Wu M, Wu D, Thiagarajan M, Wortman JR, Badger JH, Ren Q, Amedeo P, Jones KM, et al. 2006. Macronuclear genome sequence of the ciliate Tetrahymena thermophila, a model eukaryote. PLoS Biol 4: e286.

Engberg I, Pearlman RE. 1972. The amount of ribosomal RNA genes in Tetrahymena pyriformis in different physiological states. Eur J Biochem 26: 393-400.

Fenech M, Kirsch-Volders M, Natarajan AT, Surralles J, Crott JW, Parry J, Norppa H, Eastmond DA, Tucker JD, Thomas P. 2011. Molecular mechanisms of micronucleus, nucleoplasmic bridge and nuclear bud formation in mammalian and human cells. Mutagenesis 26: 125-132.

Feng W, Collingwood D, Boeck ME, Fox LA, Alvino GM, Fangman WL, Raghuraman MK, Brewer BJ. 2006. Genomic mapping of single-stranded DNA in hydroxyurea-challenged yeasts identifies origins of replication. Nat Cell Biol 8: 148155.

Flynn RL, Zou L. 2011. ATR: A master conductor of cellular responses to DNA replication stress. Trends Biochem Sci 36: 133-140.

Friedberg EC, Walker GC, Siede W, Wood RD, Schultz RA, Ellenberger T. 2006. DNA repair and mutagenesis. ASM Press, Washington, DC.

Gilbert DM. 2010. Evaluating genome-scale approaches to eukaryotic DNA replication. Nat Rev Genet 11: 673-684.

Gorovsky MA. 1973. Macro- and micronuclei of Tetrahymena pyriformis: A model system for studying the structure and function of eukaryotic nuclei. J Protozool 20: 19-25.

Grossniklaus U, Paro R. 2007. Transcriptional silencing by Polycomb group proteins. In Epigenetics (ed. CD Allis, et al.), pp. 211-230. Cold Spring Harbor Laboratory Press, Cold Spring Harbor, NY.

Groth A, Rocha W, Verreault A, Almouzni G. 2007. Chromatin challenges during DNA replication and repair. Cell 128: 721733.

Hai B, Gorovsky MA. 1997. Germ-line knockout heterokaryons of an essential $\alpha$-tubulin gene enable high-frequency gene replacement and a test of gene transfer from somatic to germ-line nuclei in Tetrahymena thermophila. Proc Natl Acad Sci 94: 1310-1315.

Hansen KH, Bracken AP, Pasini D, Dietrich N, Gehani SS, Monrad A, Rappsilber J, Lerdrup M, Helin K. 2008. A model for transmission of the H3K27me3 epigenetic mark. Nat Cell Biol 10: 1291-1300.

Harper JW, Elledge SJ. 2007. The DNA damage response: Ten years after. Mol Cell 28: 739-745.

Jacob Y, Feng S, LeBlanc CA, Bernatavichute YV, Stroud H, Cokus S, Johnson LM, Pellegrini M, Jacobsen SE, Michaels SD. 2009. ATXR5 and ATXR6 are H3K27 monomethyltransferases required for chromatin structure and gene silencing. Nat Struct Mol Biol 16: 763-768.

Jacob Y, Stroud H, Leblanc C, Feng S, Zhuo L, Caro E, Hassel C, Gutierrez C, Michaels SD, Jacobsen SE. 2010. Regulation of heterochromatic DNA replication by histone H3 lysine 27 methyltransferases. Nature 466: 987-991.

Jenuwein T, Allis CD. 2001. Translating the histone code. Science 293: $1074-1080$.
John S, Sabo PJ, Thurman RE, Sung MH, Biddie SC, Johnson TA, Hager GL, Stamatoyannopoulos JA. 2011. Chromatin accessibility pre-determines glucocorticoid receptor binding patterns. Nat Genet 43: 264-268.

Kaczanowski A, Kiersnowska M. 2011. Inactivation of a macronuclear intra-S-phase checkpoint in Tetrahymena thermophila with caffeine affects the integrity of the micronuclear genome. Protist 162: 616-636.

Karrer KM. 2012. Nuclear dualism. Methods Cell Biol 109: 29-52.

Khan SA, Nawaz MS. 2007. Direct in-gel hybridization of DNA with digoxigenin-labeled probes. Methods Mol Biol 353: 93103.

Khil PP, Smagulova F, Brick KM, Camerini-Otero RD, Petukhova GV. 2012. Sensitive mapping of recombination hotspots using sequencing-based detection of ssDNA. Genome Res 22: 957965.

Kim D, Salzberg SL. 2011. TopHat-Fusion: An algorithm for discovery of novel fusion transcripts. Genome Biol 12: R72.

Kornberg RD. 1974. Chromatin structure: A repeating unit of histones and DNA. Science 184: 868-871.

Li H, Handsaker B, Wysoker A, Fennell T, Ruan J, Homer N, Marth G, Abecasis G, Durbin R, Proc GPD. 2009. The Sequence Alignment/Map format and SAMtools. Bioinformatics 25: 2078-2079.

Liberi G, Maffioletti G, Lucca C, Chiolo I, Baryshnikova A, Cotta-Ramusino C, Lopes M, Pellicioli A, Haber JE, Foiani M. 2005. Rad51-dependent DNA structures accumulate at damaged replication forks in sgs 1 mutants defective in the yeast ortholog of BLM RecQ helicase. Genes Dev 19: 339350.

Liu Y, Mochizuki K, Gorovsky MA. 2004. Histone H3 lysine 9 methylation is required for DNA elimination in developing macronuclei in Tetrahymena. Proc Natl Acad Sci 101: 16791684.

Liu Y, Taverna S, Muratore TL, Shabanowitz J, Hunt DF, Allis CD. 2007. RNAi-dependent H3K27 methylation is required for heterochromatin formation and DNA elimination in Tetrahymena. Genes Dev 21: 1530-1545.

Lopes M, Cotta-Ramusino C, Pellicioli A, Liberi G, Plevani P, Muzi-Falconi M, Newlon CS, Foiani M. 2001. The DNA replication checkpoint response stabilizes stalled replication forks. Nature 412: 557-561.

Lopes M, Cotta-Ramusino C, Liberi G, Foiani M. 2003. Branch migrating sister chromatid junctions form at replication origins through Rad51/Rad52-independent mechanisms. Mol Cell 12: 1499-1510.

Loyola A, Bonaldi T, Roche D, Imhof A, Almouzni G. 2006. PTMs on $\mathrm{H} 3$ variants before chromatin assembly potentiate their final epigenetic state. Mol Cell 24: 309-316.

Loyola A, Tagami H, Bonaldi T, Roche D, Quivy JP, Imhof A, Nakatani Y, Dent SY, Almouzni G. 2009. The HP1 $\alpha$-CAF1SetDB1-containing complex provides H3K9me1 for Suv39mediated $\mathrm{K} 9 \mathrm{me} 3$ in pericentric heterochromatin. EMBO Rep 10: 769-775.

Luo X, Kraus WL. 2012. On PAR with PARP: Cellular stress signaling through poly(ADP-ribose) and PARP-1. Genes Dev 26: $417-432$.

MacAlpine DM, Zhang Z, Kapler GM. 1997. Type I elements mediate replication fork pausing at conserved upstream sites in the Tetrahymena thermophila ribosomal DNA minichromosome. Mol Cell Biol 17: 4517-4525.

MacNeill S. 2012. Composition and dynamics of the eukaryotic replisome: A brief overview. In The eukaryotic replisome: A guide to protein structure and function (ed. S MacNeill), pp. 1-17. Springer, New York. 
Maga G, Hubscher U. 2003. Proliferating cell nuclear antigen (PCNA): A dancer with many partners. J Cell Sci 116: 30513060.

Malkova A, Haber JE. 2012. Mutations arising during repair of chromosome breaks. Annu Rev Genet 46: 455-473.

Mar JC, Wells CA, Quackenbush J. 2011. Defining an informativeness metric for clustering gene expression data. Bioinformatics 27: 1094-1100.

Masson JY, West SC. 2001. The Rad51 and Dmc1 recombinases: A non-identical twin relationship. Trends Biochem Sci 26: 131-136.

Mechali M. 2010. Eukaryotic DNA replication origins: Many choices for appropriate answers. Nat Rev Mol Cell Biol 11: 728-738.

Mesner LD, Crawford EL, Hamlin JL. 2006. Isolating apparently pure libraries of replication origins from complex genomes. Mol Cell 21: 719-726.

Meyer E, Chalker DL. 2007. Epigenetics of ciliates. In Epigenetics (ed. CD Allis, et al.), pp. 127-150. Cold Spring Harbor Laboratory Press, Cold Spring Harbor, NY.

Miao W, Xiong J, Bowen J, Wang W, Liu Y, Braguinets O, Grigull J, Pearlman RE, Orias E, Gorovsky MA. 2009. Microarray analyses of gene expression during the Tetrahymena thermophila life cycle. PLOS ONE 4: e4429.

Minca EC, Kowalski D. 2010. Multiple Rad5 activities mediate sister chromatid recombination to bypass DNA damage at stalled replication forks. Mol Cell 38: 649-661.

Minca EC, Kowalski D. 2011. Replication fork stalling by bulky DNA damage: Localization at active origins and checkpoint modulation. Nucleic Acids Res 39: 2610-2623.

Miotto B, Struhl K. 2010. HBO1 histone acetylase activity is essential for DNA replication licensing and inhibited by Geminin. Mol Cell 37: 57-66.

Mochizuki K. 2008. High efficiency transformation of Tetrahymena using a codon-optimized neomycin resistance gene. Gene 425: 79-83.

Mochizuki K, Novatchkova M, Loidl J. 2008. DNA double-strand breaks, but not crossovers, are required for the reorganization of meiotic nuclei in Tetrahymena. J Cell Sci 121: 2148-2158.

Moldovan GL, Pfander B, Jentsch S. 2007. PCNA, the maestro of the replication fork. Cell 129: 665-679.

O'Keefe RT, Henderson SC, Spector DL. 1992. Dynamic organization of DNA replication in mammalian cell nuclei: Spatially and temporally defined replication of chromosomespecific $\alpha$-satellite DNA sequences. I Cell Biol 116: 10951110.

Palen TE, Cech TR. 1984. Chromatin structure at the replication origins and transcription-initiation regions of the ribosomal RNA genes of Tetrahymena. Cell 36: 933-942.

Petruk S, Sedkov Y, Johnston DM, Hodgson JW, Black KL, Kovermann SK, Beck S, Canaani E, Brock HW, Mazo A. 2012. TrxG and PcG proteins but not methylated histones remain associated with DNA through replication. Cell 150: 922-933.

Polo SE, Jackson SP. 2011. Dynamics of DNA damage response proteins at DNA breaks: A focus on protein modifications. Genes Dev 25: 409-433.

Qin ZS, Yu J, Shen J, Maher CA, Hu M, Kalyana-Sundaram S, Chinnaiyan AM. 2010. HPeak: An HMM-based algorithm for defining read-enriched regions in ChIP-seq data. BMC Bioinformatics 11: 369.

Raynaud C, Sozzani R, Glab N, Domenichini S, Perennes C, Cella R, Kondorosi E, Bergounioux C. 2006. Two cell-cycle regulated SET-domain proteins interact with proliferating cell nuclear antigen (PCNA) in Arabidopsis. Plant J 47: 395407.
Reichard P. 1988. Interactions between deoxyribonucleotide and DNA synthesis. Annu Rev Biochem 57: 349-374.

Salamone MF, Nachtwey DS. 1979. Formation and fate of extranuclear chromatin bodies in Tetrahymena. J Protozool 26: 227-231.

San Filippo J, Sung P, Klein H. 2008. Mechanism of eukaryotic homologous recombination. Annu Rev Biochem 77: 229257.

Schwartz EK, Heyer WD. 2011. Processing of joint molecule intermediates by structure-selective endonucleases during homologous recombination in eukaryotes. Chromosoma 120: 109-127.

Sclafani RA, Holzen TM. 2007. Cell cycle regulation of DNA replication. Annu Rev Genet 41: 237-280.

Segal E, Widom J. 2009. Poly(dA:dT) tracts: Major determinants of nucleosome organization. Curr Opin Struct Biol 19: 65-71.

Sfeir A, Kosiyatrakul ST, Hockemeyer D, MacRae SL, Karlseder J, Schildkraut CL, de Lange T. 2009. Mammalian telomeres resemble fragile sites and require TRF1 for efficient replication. Cell 138: 90-103.

Shen X, Liu Y, Hsu YJ, Fujiwara Y, Kim J, Mao X, Yuan GC, Orkin SH. 2008. EZH1 mediates methylation on histone $\mathrm{H} 3$ lysine 27 and complements EZH2 in maintaining stem cell identity and executing pluripotency. Mol Cell 32: 491-502.

Skovorodkin IN, Zassoukhina IB, Hojak S, Ammermann D, Gunzl A. 2001. Minichromosomal DNA replication in the macronucleus of the hypotrichous ciliate Stylonychia lemnae is independent of chromosome-internal sequences. Chromosoma 110: 352-359.

Solimando L, Luijsterburg MS, Vecchio L, Vermeulen W, van Driel R, Fakan S. 2009. Spatial organization of nucleotide excision repair proteins after UV-induced DNA damage in the human cell nucleus. J Cell Sci 122: 83-91.

Strahl BD, Allis CD. 2000. The language of covalent histone modifications. Nature 403: 41-45.

Sweet MT, Allis CD. 1998. Culture and biochemical analysis of cells. In Cells: a laboratory manual (ed. DL Spector, et al.). Cold Spring Harbor Laboratory Press, Cold Spring Harbor, NY.

Tanaka S, Araki H. 2010. Regulation of the initiation step of DNA replication by cyclin-dependent kinases. Chromosoma 119: $565-574$

Tardat M, Brustel J, Kirsh O, Lefevbre C, Callanan M, Sardet C, Julien E. 2010. The histone H4 Lys 20 methyltransferase PRSet7 regulates replication origins in mammalian cells. Nat Cell Biol 12: 1086-1093.

Vogelauer M, Rubbi L, Lucas I, Brewer BJ, Grunstein M. 2002. Histone acetylation regulates the time of replication origin firing. Mol Cell 10: 1223-1233.

Weinert T, Kaochar S, Jones H, Paek A, Clark AJ. 2009. The replication fork's five degrees of freedom, their failure and genome rearrangements. Curr Opin Cell Biol 21: 778-784.

Wysocka J. 2006. Identifying novel proteins recognizing histone modifications using peptide pull-down assay. Methods 40: 339-343.

Xiong J, Lu X, Zhou Z, Chang Y, Yuan D, Tian M, Wang L, Fu C, Orias E, Miao W. 2012. Transcriptome analysis of the model protozoan, Tetrahymena thermophila, using Deep RNA sequencing. PLOS ONE 7: e30630.

Xu J, Yanagisawa Y, Tsankov AM, Hart C, Aoki K, Kommajosyula N, Steinmann KE, Bochicchio J, Russ C, Regev A, et al. 2012a. Genome-wide identification and characterization of replication origins by deep sequencing. Genome Biol 13: R27.

Xu M, Wang W, Chen S, Zhu B. 2012b. A model for mitotic inheritance of histone lysine methylation. EMBO Rep 13: $60-67$. 
Yakisich JS, Sandoval PY, Morrison TL, Kapler GM. 2006. TIF1 activates the intra-S-phase checkpoint response in the diploid micronucleus and amitotic polyploid macronucleus of Tetrahymena. Mol Biol Cell 17: 5185-5197.

Yao MC, Kimmel AR, Gorovsky MA. 1974. A small number of cistrons for ribosomal RNA in the germinal nucleus of a eukaryote, Tetrahymena pyriformis. Proc Natl Acad Sci 71: 3082-3086.

Yu L, Gorovsky MA. 1997. Constitutive expression, not a particular primary sequence, is the important feature of the $\mathrm{H} 3$ replacement variant hv2 in Tetrahymena thermophila. Mol Cell Biol 17: 6303-6310.

Yu Y, Song C, Zhang Q, DiMaggio PA, Garcia BA, York A, Carey MF, Grunstein M. 2012. Histone H3 lysine 56 methylation regulates DNA replication through its interaction with PCNA. Mol Cell 46: 7-17.

Yusufzai T, Kadonaga JT. 2011. Branching out with DNA helicases. Curr Opin Genet Dev 21: 214-218.

Zhang Z, Macalpine DM, Kapler GM. 1997. Developmental regulation of DNA replication: Replication fork barriers and programmed gene amplification in Tetrahymena thermophila. Mol Cell Biol 17: 6147-6156.

Zhang C, Molascon AJ, Gao S, Liu Y, Andrews PC. 2013. Quantitative proteomics reveals that the specific methyltransferases Txrlp and Ezl2p differentially affect the mono-, di- and tri-methylation states of histone H3 lysine 27. Mol Cell Proteomics 12: 1678-1688.

Zou L, Elledge SJ. 2003. Sensing DNA damage through ATRIP recognition of RPA-ssDNA complexes. Science 300: 15421548. 


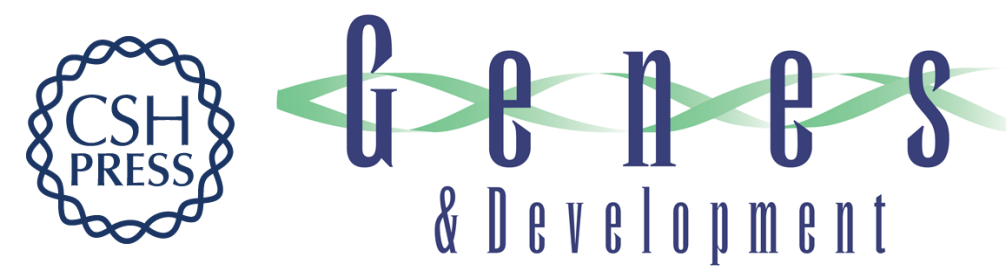

\section{Impaired replication elongation in Tetrahymena mutants deficient in histone $\mathrm{H} 3$ Lys 27 monomethylation}

Shan Gao, Jie Xiong, Chunchao Zhang, et al.

Genes Dev. 2013, 27: originally published online July 24, 2013

Access the most recent version at doi:10.1101/gad.218966.113

\section{Supplemental http://genesdev.cshlp.org/content/suppl/2013/07/17/gad.218966.113.DC1 Material}

References This article cites 93 articles, 23 of which can be accessed free at: http://genesdev.cshlp.org/content/27/15/1662.full.html\#ref-list-1

\section{License}

Email Alerting

Receive free email alerts when new articles cite this article - sign up in the box at the top Service

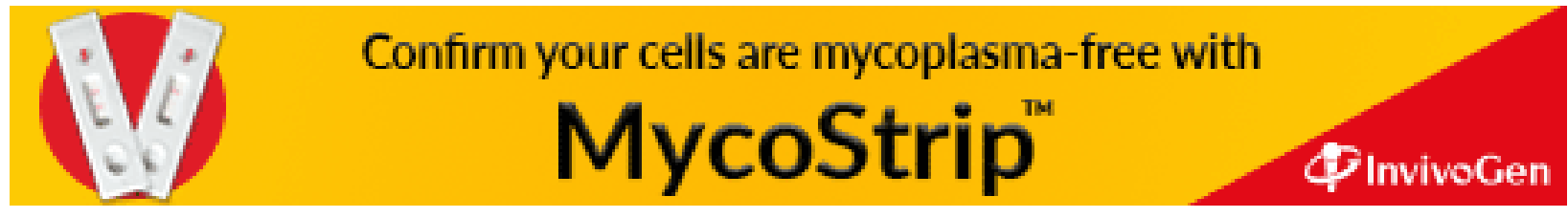

